\title{
Colloquium: The spin-incoherent Luttinger liquid
}

\author{
Gregory A. Fiete* \\ Department of Physics, California Institute of Technology, MC 114-36, Pasadena, \\ California 91125, USA and Kavli Institute for Theoretical Physics, University of California, \\ Santa Barbara, California 93106, USA
}

(Published 13 July 2007)

\begin{abstract}
In contrast to the well-known Fermi-liquid theory of three dimensions, interacting one-dimensional and quasi-one-dimensional systems of fermions are described at low energy by an effective theory known as Luttinger liquid theory. This theory is expressed in terms of collective many-body excitations that show exotic behavior such as spin-charge separation. Luttinger liquid theory is commonly applied on the premise that "low energy" describes both the spin and charge sectors. However, when the interactions in the system are very strong, as they typically are at low particle densities, the ratio of spin to charge energy may become exponentially small. It is then possible at very low temperatures for the single-spin excitation energy to be low compared to the characteristic single excitation charge energy, but still high compared to the characteristic spin energy. This energy window of near ground-state charge degrees of freedom but highly thermally excited spin degrees of freedom is called a spin-incoherent Luttinger liquid. The spin-incoherent Luttinger liquid exhibits a higher degree of universality than the Luttinger liquid and its properties are qualitatively distinct. In this Colloquium some recent theoretical developments in the field are detailed and experimental indications of such a regime in gated semiconductor quantum wires are described.
\end{abstract}

DOI: 10.1103/RevModPhys.79.801

PACS number(s): 71.10.Pm, 71.27.+a, 73.21.-b

\section{CONTENTS}

I. Introduction

II. Fluctuating Wigner Solid Model
A. Effective Hamiltonian
B. "Superuniversal" spin physics
C. Effective low-energy spin Hamiltonian

III. Particle Nonconserving Operators

A. Single-particle Green's function

1. $\mathcal{G}_{\sigma}(x, \tau)$ for large $x$

2. $\mathcal{G}_{\sigma}(x, \tau)$ for small $x$

3. Tunneling density of states

4. Finite magnetic field

B. Fermi-edge singularity

C. Spin-incoherent effects in transport

IV. Particle Conserving Operators

A. Mapping to spinless electrons in the spin-incoherent Luttinger liquid

B. Spin-charge coupling in Wigner solid model

C. Description of the effective density

D. $2 k_{F}$ density correlations

E. Coulomb drag, noise, and dephasing

V. Experimental Evidence for Spin Incoherence

A. Momentum-resolved tunneling at low particle density

B. Theoretical support for the spin-incoherent Luttinger liquid in experiment

1. Exact diagonalization for few electrons

2. Comparison with spin-incoherent Luttinger liquid theory

801

803

803

804

804

805

805

806

807

808

808

809

810

811

812

812

813

813

813

814

814

817

817

818

*Electronic address: fiete@caltech.edu
VI. Outlook and Open Issues

819

Acknowledgments

819

References

820

\section{INTRODUCTION}

This Colloquium is about the properties of strongly interacting fermions in one spatial dimension in a certain window of energies, but before I discuss the details, it is worthwhile to recall what we "know" about interacting fermions. First, fermions obey Pauli exclusion statistics - no two particles can occupy the same state, i.e., have the same set of quantum numbers. The simplest case to consider is a noninteracting system of fermions. Often one is interested in the ground state and the nature of the lowest-lying excitations above the ground state. If we have noninteracting fermions, the problem is a relatively simple one to solve. We just find all the eigenstates of a single-particle Hamiltonian and then fill them (with one particle each because of the Pauli principle) starting from the lowest energy state until all $N$ electrons in the system occupy a state. By construction this is the ground state. If $N$ is large, as it is for the number of electrons in a metal, for example, one refers to the set of filled states as a "Fermi sea" and the "top" of the sea is called a "Fermi surface," which in one dimension actually consists of only two points in momentum space. This sea structure is a direct consequence of the Pauli exclusion principle and our noninteracting particle assumption. The lowest-lying excitations are also easy to find: We take a particle near the "surface" and move it above the surface (since it cannot be moved below because all those states are filled by construction). This process leaves a "hole" in 
the Fermi sea and a particle excited above the Fermi sea. Naturally, such excitations are called particle-hole excitations.

Now suppose we make the system more realistic by remembering that real fermions interact. The effect of these interactions remarkably turns out to depend on the spatial dimension being considered. In three dimensions the Fermi surface survives and the low-energy particle and hole excitations are very much like those in the noninteracting case only with a renormalization of their mass, and they acquire a lifetime inversely proportional to the square of the excitation energy relative to the Fermi-surface energy. The theory describing this situation is called Fermi-liquid theory and it has been successfully applied to liquid ${ }^{3} \mathrm{He}$ and many metals (Vollhardt, 1984).

On the other hand, in one spatial dimension, the Fermi surface does not survive (in the sense that no lowenergy excitations of single-particle character exist, although the Fermi wave vector $k_{F}$ remains a special value) and a new state of matter is born-the Luttinger liquid. The Luttinger liquid contains only collective many-body excitations which can be separated into spin and charge sectors that propagate with different collective-mode velocities. Note that this is remarkably different from the intuition we have from a single electron where the spin and charge are tied together. The details of how and why spin-charge separation occur are well understood and there are a number of sources on the topic. ${ }^{1}$ We will not need those technical details here.

The easiest way to understand the physics of the spinincoherent Luttinger liquid is to see what goes wrong with a Luttinger liquid when the temperature is large compared to the single excitation spin energy, but still small compared to the single excitation energy of charge. ${ }^{2}$ For that we need to be familiar with a few of the central results of Luttinger liquid theory itself. To illustrate the consequences of spin-charge separation and get a flavor of the differences between the Luttinger liquid and the spin-incoherent Luttinger liquid we use the single-particle Green's function. Later we will discuss what goes wrong with a Luttinger liquid when the temperature is higher than the spin energy.

The single-particle Green's function in imaginary time $\tau=i t$ is $\mathcal{G}_{\sigma}(x, \tau)=-\left\langle T_{\tau} \psi_{\sigma}(x, \tau) \psi_{\sigma}^{\dagger}(0,0)\right\rangle$ where $\psi_{\sigma}^{\dagger}\left(\psi_{\sigma}\right)$ is the creation (annihilation) operator for a fermion of spin projection $\sigma$ along the $z$ axis and $T_{\tau}$ is the $\tau$ ordering operator. For a Luttinger liquid, one finds ${ }^{3}$

\footnotetext{
${ }^{1}$ See, for example, Voit (1995); Chang (2003); Giamarchi (2004).

${ }^{2}$ This wide separation of spin and charge energy scales requires strong interactions and is discussed in Sec. II.

${ }^{3}$ The formula below assumes $\mathrm{SU}(2)$ symmetry in the spin sector.
}

$$
\mathcal{G}_{\sigma}^{\mathrm{LL}}(x, \tau) \sim \frac{1 / \sqrt{v_{s} \tau-i x}}{\left(x^{2}+v_{c}^{2} \tau^{2}\right)^{\gamma_{K_{c}}}} \frac{e^{i k_{F} x}}{\sqrt{v_{c} \tau-i x}}+\text { c.c. },
$$

where $\gamma_{K_{c}}=\left(K_{c}+K_{c}^{-1}-2\right) / 8 \geqslant 0, v_{s}$ is the spin velocity, $v_{c}$ is the charge velocity, and the Fermi wave vector $k_{F} \equiv \pi /(2 a)$ with $a$ the interparticle spacing. The singleparticle Green's function (1) clearly illustrates the effects of spin-charge separation when $v_{s} \neq v_{c}$ : when a particle is added at $(0,0)$ the charge and spin components propagate with different velocities. Physically, this occurs because the Hamiltonian for the interacting onedimensional (1D) system separates at low energies to $H=H_{s}+H_{c}$ where $H_{s}$ is the spin Hamiltonian and $H_{c}$ is the charge Hamiltonian. Note the branch cut structure of the singularities in Eq. (1). The absence of a simple pole is related to the absence of quasiparticle excitations in a Luttinger liquid. When fermions are noninteracting one has $K_{c}=1$ and $v_{s}=v_{c}$, and the noninteracting Green's function is recovered from Eq. (1) along with the simple pole structure characteristic of quasiparticle excitations.

By contrast, in the spin-incoherent case (defined as the regime where the spin energy $E_{\text {spin }} \approx \hbar v_{s} / a \ll k_{B} T$ $\ll \hbar v_{c} / a \approx E_{\text {charge }}$ where $T$ is the temperature, $k_{B}$ is Boltzmann's constant, and $\hbar$ is Planck's constant divided by $2 \pi$ ) we have

$$
\mathcal{G}_{\sigma}^{\operatorname{SILL}}(x, \tau) \sim \frac{e^{-2 k_{F}|x|(\ln 2 / \pi)}}{\left(x^{2}+v_{c}^{2} \tau^{2}\right)^{\Delta_{K}}} \frac{e^{i\left(2 k_{F} x-\varphi_{K_{c}}^{+}\right)}}{v_{c} \tau-i x}+\text { c.c. },
$$

where $\Delta_{K_{c}} \neq \gamma_{K_{c}}$ and $\varphi_{K_{c}}$ are given in Sec. III. In the spin-incoherent regime the Hamiltonian is still spincharge separated and many features of the Green's function (2) are reminiscent of Eq. (1). However, there are important differences. First, note the exponential decay in Eq. (2) which replaces the square-root branch cut from the spin mode in the Luttinger liquid. The exponential factor is distinct from the Luttinger liquid case (1) in which only power laws appear and can be said to put the spin-incoherent Luttinger liquid in a different universality class from the Luttinger liquid. Second, note that the spin velocity $v_{s}$ has dropped out of Eq. (2) implying that the spin degrees of freedom are nonpropagating. It turns out that the absence of $v_{s}$ in the Green's function is a specific case of a more general "superuniversal" spin physics that occurs in the spin-incoherent regime in which no parameter of the spin Hamiltonian enters the correlation functions. As a result, the correlations are completely independent of $H_{s}$ and this implies a higher degree of universality in the spin-incoherent Luttinger liquid than in the Luttinger liquid since $H_{s}$ will not affect any observable properties of the system in this regime. Third, note the shift $k_{F} \rightarrow 2 k_{F}$ in the oscillating wave vector of the Green's function. This shift comes from the spin incoherence itself and can also be seen in the momentum distribution function $n(k)$ (Cheianov et al., 2005).

Another quantity which illustrates a remarkable difference between the Luttinger liquid and the spin incoherent Luttinger liquid is the frequency dependence 
of the tunneling density of states (which can be derived from the imaginary part of the retarded Green's function). For a Luttinger liquid one finds

$$
A^{\mathrm{LL}}(\omega) \sim \omega^{\left(K_{c}+K_{c}^{-1}-2\right) / 4},
$$

and for a spin-incoherent Luttinger liquid

$$
A^{\operatorname{SILL}}(\omega) \sim \omega^{\left(1 / 4 K_{C}\right)-1} / \sqrt{|\ln (\omega)|} .
$$

As with the single-particle Green's function there is a qualitative difference in the tunneling density of states: The Luttinger liquid always exhibits a suppression of the tunneling as $\omega \rightarrow 0$, while the spin-incoherent Luttinger liquid will show a divergence ${ }^{4}$ for decreasing $\omega$ if $K_{c}$ $>1 / 4$, which will be the case for many systems exhibiting the spin-incoherent Luttinger liquid phase. In addition there are logarithmic corrections which turn out to appear quite generally in a whole class of quantities (see Sec. III). There are many other distinct signatures of the spin-incoherent Luttinger liquid that appear in transport, interference experiments, tunneling, the Fermiedge singularity, Coulomb drag, and noise that are detailed in this Colloquium.

It is worth noting that while certain aspects of the spin-incoherent equal-time Green's function were computed by Berkovich (1991), the full space-time dependence of the spin-incoherent Luttinger liquid regime appears to have been first studied by Matveev (2004a) as a possible explanation for the 0.7 conductance feature in quantum point contacts and by Cheianov and Zvonarev (2004a) in which they investigated the single-particle Green's function in the spin-incoherent regime of the infinite- $U$ Hubbard model. All of these works have proved invaluable in establishing the field and to subsequent progress in it.

In the remainder of the Colloquium I will develop some of the relevant theory to describe the spinincoherent Luttinger liquid. Since the spin-incoherent Luttinger liquid requires very strong interactions, a useful starting point is to model fermions as a fluctuation Wigner solid. This approach provides a convenient conceptual framework for understanding the most important aspects of the spin-incoherent Luttinger liquid. For reasons that will become clear as we proceed, the features of the spin-incoherent Luttinger liquid fall into two categories depending on whether the relevant correlation functions are particle nonconserving (such as the single-particle Green's function) or particle conserving (such as the density-density correlation function). I will cover these issues in detail and illustrate the main points with examples in each case. The Colloquium will close with a brief discussion of experimental indications of the spin-incoherent Luttinger liquid in semiconductor quantum wires and some of the outstanding problems in the field.

\footnotetext{
${ }^{4}$ The divergence will only be seen for $\hbar \omega \gtrsim k_{B} T$. For smaller $\omega$ the divergence is cut off (Matveev et al., 2007).
}

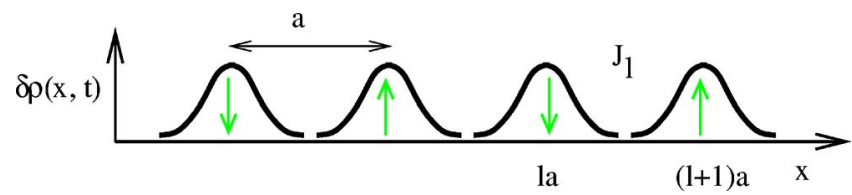

FIG. 1. (Color online) Schematic of a fluctuating Wigner solid model. Shown is a snapshot in time of the density modulations $\delta \rho(x, t)$ of a fluctuating Wigner solid in one dimension of mean particle spacing $a$. A cartoon of the antiferromagnetic spin sector is shown with the light arrows representing spin orientation on the Wigner solid sites given by la. There is an antiferromagnetic exchange $J_{l}$ between the $l$ th and $(l+1)$ th sites. The lattice has a stiffness characterized by the frequency $\omega_{0} \gg J_{l} / \hbar$.

\section{FLUCTUATING WIGNER SOLID MODEL}

The spin-incoherent Luttinger liquid requires $E_{\text {spin }}$ $\ll E_{\text {charge }}$ and this separation of spin and charge energy scales only occurs for strong interactions. The discussion in this section attempts to explain why strong interactions are necessary and how to simply model this situation. Consider a one-dimensional gas of spin- $1 / 2$ fermions. The typical kinetic energy K.E. $\sim \frac{\hbar^{2} k_{F}^{2}}{2 m^{*}}$ where $m^{*}$ is the effective mass, and the typical potential energy P.E. $\sim \frac{e^{2}}{\epsilon a}$ where $\epsilon$ is the dielectric constant and $e$ is the charge of the fermion. Since $k_{F} \propto n=1 / a$, we see that $\frac{\text { P.E. }}{\text { K.E. }} \sim \frac{1}{n a_{B}} \sim r_{s}$, where $a_{B} \equiv \frac{\epsilon \hbar^{2}}{m^{*} e^{2}}$ is the Bohr radius of the material and $r_{s} \equiv 1 /\left(2 n a_{B}\right)$ is a dimensionless parameter describing how strong the potential energy is relative to the kinetic energy. Obviously, the larger $r_{s}$ is, the more solidlike the electron gas (see Fig. 1 for a schematic). The solid phase is actually believed to be obtained in two and three dimensions for sufficiently large $r_{s}$ (Tanatar and Ceperley, 1989). However, in one dimension the quantum fluctuations are strong enough to destroy the long-range order, even for long-range Coulomb interactions (Schulz, 1993). Nevertheless, a "fluctuating" Wigner solid still provides a useful starting point for strongly interacting fermions in one dimension and such a model will be used throughout the remainder of this Colloquium.

\section{A. Effective Hamiltonian}

A fluctuating Wigner solid is mathematically equivalent to a harmonic chain ${ }^{5}$ with Hamiltonian

$$
H_{\text {chain }}=\sum_{l=1}^{N} \frac{p_{l}^{2}}{2 m^{*}}+\frac{m^{*} \omega_{0}^{2}}{2}\left(u_{l+1}-u_{l}\right)^{2},
$$

where $p_{l}$ is the momentum of the $l$ th fermion, $u_{l}$ is the displacement from equilibrium of the $l$ th fermion, and

\footnotetext{
${ }^{5}$ An alternative description of a Wigner solid has been discussed by Novikov (2005a, 2005b).
} 
$\omega_{0}$ is the frequency of local fermion displacements. ${ }^{6}$ The positions of fermions along the chain are given by

$$
x_{l}=l a+u_{l}
$$

where $a$ is the mean spacing of fermions as before. Since we are dealing with a quantum system, we can canonically quantize the harmonic chain by imposing $\left[u_{l}, p_{l^{\prime}}\right]$ $=i \hbar \delta_{l l^{\prime}}$.

Fermions also have a spin degree of freedom, and in one dimension very general considerations (Lieb and Mattis, 1962) result in antiferromagnetic interactions. The simplest situation is when the exchange is of the nearest-neighbor type in the Wigner solid. In this case, the Hamiltonian of the spin sector takes the form

$$
H_{s}=\sum_{l} J_{l} \vec{S}_{l} \cdot \vec{S}_{l+1},
$$

where $J_{l}$ is the nearest-neighbor exchange between the $l$ th and $(l+1)$ th sites in the lattice (see Fig. 1). The spin Hamiltonian (7) is the generic low-density form for SU(2) symmetric interactions regardless of whether the interactions between electrons are zero range, as in the Hubbard model, or long-range unscreened Coulomb interactions (Ogata and Shiba, 1990; Matveev, 2004b). Moreover, in the strongly interacting regime assumed here, one can argue on the general grounds that the exchange energy $J_{l}$ is exponentially suppressed relative to $\hbar \omega_{0}$, the characteristic charge energy. Various approximations for $J_{l}$ have been discussed. ${ }^{7}$ The main physical point is that in one dimension when the interactions are strong, two particles must tunnel through each other in order to "exchange." It is just these processes that set the scale for $J_{l}$ which is exponentially small relative to $\hbar \omega_{0}$ because of the tunneling processes involved. ${ }^{8}$

In this Colloquium we will only be interested in energy scales small compared to the characteristic charge energy $\hbar \omega_{0}$, so we would like to find a simpler, lowenergy form for Eq. (5). To do so, we take a continuum limit and convert the sum over $l$ to an integral over $x$ (Schulz, 1993; Giamarchi, 2004; Matveev, 2004b). In order to make the low-energy Hamiltonian look like the Hamiltonian familiar from Luttinger liquid theory, we also rescale the variables $u_{l} \rightarrow u(x) \equiv a \frac{\overline{2}}{\pi} \theta_{c}(x)$ and $p_{l}$ $\rightarrow p(x) \equiv \frac{\hbar}{a \overline{2}} \partial_{x} \phi_{c}(x)$ which then satisfy $\left[u(x), p\left(x^{\prime}\right)\right]$ $=\hbar\left[\theta_{c}(x), \partial_{x^{\prime}} \phi_{c}\left(x^{\prime}\right)\right]=i \hbar \pi \delta\left(x-x^{\prime}\right)$ which is just the continuum version of $\left[u_{l}, p_{l^{\prime}}\right]=i \hbar \delta_{l l^{\prime}}$. The resulting lowenergy charge Hamiltonian is

\footnotetext{
${ }^{6}$ When the physical system of interest is electrons in a quantum wire, $\omega_{0}$ will depend on the density, the width of the quantum wires, the dielectric constant of the material, and the distance to a nearby metallic gate (Glazman et al., 1992; Häusler et al., 2002).

${ }^{7}$ See, for example, Häusler (1996); Matveev (2004b); Fogler and Pivovarov (2005); Klironomos et al. (2005).

${ }^{8}$ Although the physics we discuss here assumes low density, strongly correlated physics can also occur in very thin quantum wires at higher density (Fogler, 2005a, 2005b; Kindermann, 2007).
}

$$
H_{c}=\hbar v_{c} \int \frac{d x}{2 \pi}\left(\frac{1}{K_{c}}\left[\partial_{x} \theta_{c}(x)\right]^{2}+K_{c}\left[\partial_{x} \phi_{c}(x)\right]^{2}\right),
$$

where $v_{c}=\omega_{0} a$ and $K_{c}=\frac{\pi \hbar}{2 a m v_{c}}$. For the rest of this Colloquium, we will exclusively use Eq. (8) to describe the charge sector of the spin-incoherent Luttinger liquid as we are only interested in energies which are low compared to the characteristic charge energy, $E_{\text {charge }} \approx \hbar \omega_{0}$. The full Hamiltonian for all energies of interest is then

$$
H=H_{c}+H_{s},
$$

with $H_{c}$ given by Eq. (8) and $H_{s}$ given by Eq. (7).

\section{B. "Superuniversal" spin physics}

Having obtained the relevant Hamiltonian we are now in a position to compute expectation values,

$$
\langle A\rangle=\frac{1}{Z} \operatorname{Tr}\left[e^{-\beta H} A\right],
$$

where $A$ is any operator, $Z \equiv \operatorname{Tr}\left[e^{-\beta H}\right]$ is the partition function, and $\beta=\left(k_{B} T\right)^{-1}$. Since the Hamiltonian separates into spin and charge pieces, the trace can be evaluated for each independently. Consider the trace over the spin degrees of freedom. ${ }^{9}$ In the spin-incoherent regime we have $E_{\text {spin }} \ll k_{B} T$ which implies $e^{-\beta H_{s}} \approx 1$. Therefore the spin Hamiltonian completely drops out of the trace. This is precisely the reason why no parameter of the spin Hamiltonian appeared in the single-particle Green's function (2), and one can see here that it is a very general feature of the spin-incoherent regime that expectation values (and correlation functions derived from them) are completely independent of $H_{s}$. In this sense the spin physics exhibited in the regime $E_{\text {spin }} \ll k_{B} T$ is "superuniversal" and one can see, perhaps trivially, that the spin-incoherent Luttinger liquid has a higher degree of universality than a Luttinger liquid whose correlation functions will depend on $H_{s}$. Note that even if $H_{s}$ is gapped the correlations will remain unaffected provided $E_{\text {spin }} \ll k_{B} T$.

\section{Effective low-energy spin Hamiltonian}

Before moving on to the next section, we note that the low-energy (for $k_{B} T \ll E_{\text {spin }}$ ) form of Eq. (7) is given by

$$
H_{s}^{\text {low }}=\hbar v_{s} \int \frac{d x}{2 \pi}\left(\frac{1}{K_{s}}\left[\partial_{x} \theta_{s}(x)\right]^{2}+K_{s}\left[\partial_{x} \phi_{s}(x)\right]^{2}\right),
$$

where $v_{s}=J a / \hbar$ is the spin velocity, and $K_{s}=1$ for an $\mathrm{SU}(2)$ symmetric spin sector. The bosonic fields satisfy $\left[\theta_{\alpha}(x), \phi_{\beta}(y)\right]=-i \frac{1}{2} \pi \delta_{\alpha, \beta} \operatorname{sgn}(x-y)$, where $\alpha, \beta=s$ or $c$. In later sections we will make use of the low-energy form (11) when discussing how the Luttinger liquid breaks down when $k_{B} T$ approaches $E_{\text {spin }}$ from below. Any Lut-

\footnotetext{
${ }^{9}$ For simplicity assume that $J_{l}=J$ independent of $l$, i.e., there is no magnetoelastic coupling. In Sec. IV we see that there are effects resulting from the $l$ dependence of $J_{l}$.
} 
tinger liquid (with $v_{s} / v_{c} \ll 1$ ) at $T=0$ becomes a spinincoherent Luttinger liquid when $E_{\text {spin }} \ll k_{B} T \ll E_{\text {charge }}$.

\section{PARTICLE NONCONSERVING OPERATORS}

Armed with the Hamiltonian (9) one can calculate the properties of the spin-incoherent Luttinger liquid. In this section, we will focus on results obtained from correlation functions of particle nonconserving operators, such as the particle creation or annihilation operator that appears in the single-particle Green's function. These correlation functions will be evaluated in a first quantized path-integral representation which makes clear why there is a distinction between particle conserving and nonconserving operators in the spin-incoherent regime.

\section{A. Single-particle Green's function}

The single-particle Green's function in imaginary time $\tau=i t$ is

$$
\mathcal{G}_{\sigma}(x, \tau)=-\frac{1}{Z} \operatorname{Tr}\left[e^{-\beta H} \psi_{\sigma}(x, \tau) \psi_{\sigma}^{\dagger}(0,0)\right], \quad \tau>0,
$$

where $\psi_{\sigma}^{\dagger}\left(\psi_{\sigma}\right)$ is the creation (annihilation) operator for a fermion of spin projection $\sigma$ along the $z$ axis and $Z$ $\equiv \operatorname{Tr}\left[e^{-\beta H}\right]$ is the partition function as before. We evaluate the trace using a first quantized path-integral representation of $\mathcal{G}_{\sigma}(x, \tau)$ (Fiete and Balents, 2004). In this representation the trace is an integral over world lines (paths) of particle trajectories. ${ }^{10}$ Each configuration is weighted by a Euclidean action describing the "deformation" of world lines (see Fig. 2) and a statistical factor reflecting the sign of the permutation of the fermions.

In evaluating the trace over the spin and charge degrees of freedom it is convenient to make maximum use of the hierarchy of energies relevant to the spinincoherent Luttinger liquid: $E_{\text {spin }} \ll k_{B} T \ll E_{\text {charge. }}$. A crucial part of our method of evaluating the trace is to make a noncrossing approximation in the space of world lines. As explained earlier, $E_{\text {spin }}$ is small because particle exchanges are rare due to the fact that particles must tunnel through each other when the interactions are strong. Given $E_{\text {spin }}$, this argument can then be turned around: the typical exchange (crossing) time $t_{\text {exch }}$ $\sim \hbar / E_{\text {spin }}$ is very long. In particular, the exchange time is much longer than the thermal coherence time $t_{\mathrm{coh}}$ $\sim \hbar / k_{B} T=\hbar \beta$ which is the amount of time a particle has to propagate in the imaginary time path-integral formulation. Therefore on the time scale of the coherence time, no particles will exchange their positions. We call this the noncrossing approximation and it restricts the possible world-line trajectories to the types shown in

\footnotetext{
${ }^{10}$ There are remarkable similarities between the analysis here and that for the gapped phases of 1D spin chains (Sachdev and Damle, 1997; Damle and Sachdev, 1998, 2005; Rapp and Zarand, 2006).
}

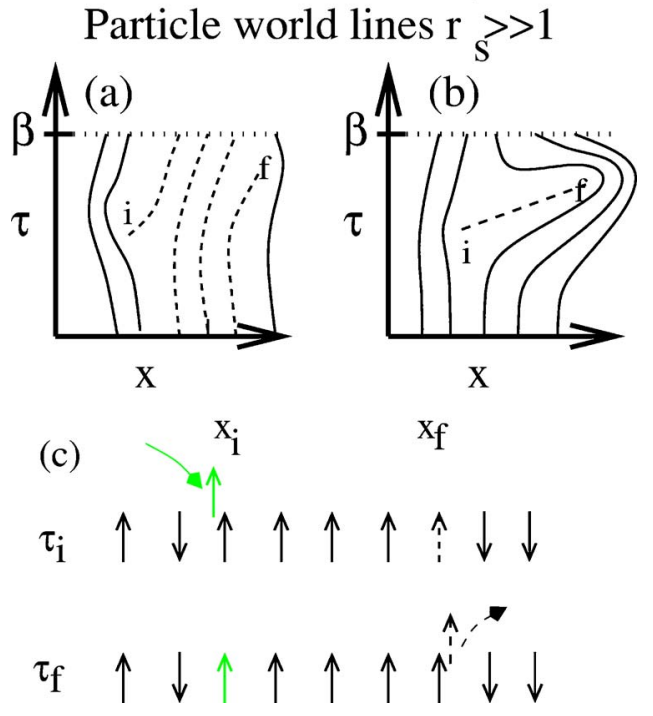

FIG. 2. (Color online) World lines for strongly interacting onedimensional spin- $S$ fermions for $E_{\text {spin }} \ll k_{B} T \ll E_{\text {charge }}$ in which the noncrossing approximation is made. Particle trajectories in space and imaginary time are shown as curved lines. Dashed lines represent the world line paths for creating a particle and removing it for large $x=x_{f}-x_{i}, \tau=\tau_{f}-\tau_{i}$. The solid lines represent trajectories of other particles. Because of the large action cost associated with the trajectories in (b), at low energies (relative to $E_{\text {charge }}$ ) a process like that shown in (a) where world lines wrap around from $\tau=\beta$ to $\tau=0$ will dominate. Such a process, however, requires that all dashed world lines have the same spin. For $E_{\text {spin }} \ll k_{B} T$ in zero magnetic field, this occurs with probability $(2 S+1)^{-|N|}$. Physically, the process shown in (a) is equivalent to adding an electron at $\left(x_{i}, \tau_{i}\right)$ then pushing all electrons to the right and removing the electron at $\left(x_{f}, \tau_{f}\right)$ as shown in (c). In order for the final spin configuration to look the same as the original one, all spins must be aligned between the initial and final points.

Fig. 2. It is worth emphasizing that this argument is completely general and exploits no feature of $H_{s}$ other than its characteristic energy $E_{\text {spin }}$.

Having restricted the class of world lines that contribute to the Green's function in the spin-incoherent Luttinger liquid, a further subset can be selected that provides the dominant contribution to the Green's function. These are the configurations shown in Fig. 2(a) in which the trajectories of the added particle effectively wrap around the imaginary time torus. These contributions minimize the action of the charge sector while obeying the noncrossing approximation. Contributions such as those shown in Fig. 2(b) are exponentially suppressed in weight relative to those shown in Fig. 2(a). In order for the trajectories to wrap around the imaginary time torus, the spin configuration of each world line between the initial and final point must be the same. For a spin- $S$ fermion, this will occur with probability $(2 S$ $+1)^{-|N|}$ where $N$ is the number of world lines between the initial and final position. Additionally, there is a permutation factor $(-1)^{N}$ coming from the Fermi statistics. Selecting this class of trajectories thus constrains the spins and is therefore equivalent to computing the trace 
over the spin degrees of freedom. The resulting dynamics therefore becomes effectively spinless and we are left with

$$
\begin{aligned}
\mathcal{G}_{\sigma}(x, \tau) \sim & \sum_{m=-\infty}^{\infty}\left\langle\delta(m-N(x, \tau))(-1)^{m}\right. \\
& \left.\times(2 S+1)^{-|m|} e^{i[\phi(x, \tau)-\phi(0,0)]}\right\rangle,
\end{aligned}
$$

where the number of world lines $N(x, \tau)$ is allowed to fluctuate dynamically and the factor $e^{i \phi(x, \tau)}\left(e^{-i \phi(x, \tau)}\right)$ annihilates (creates) a particle at $(x, \tau)$. One must sum over all possible numbers of particles $m$ between the initial and final points.

The remaining expectation value is computed over the charge degrees of freedom alone. It is at this stage that we make use of the second part of the spin-incoherent Luttinger liquid energy hierarchy, $k_{B} T \ll E_{\text {charge }}$, to compute the remaining average for $T \rightarrow 0$ using Eq. (8). In order to compute the average over the charge degrees of freedom the number of particles $N(x, \tau)$ must be related to the fluctuating charge variables of the Hamiltonian (8). This relation is obtained simply by integrating over the fluctuating density between the initial and final space-time points (Fiete and Balents, 2004),

$$
\begin{aligned}
N(x, \tau) & =n x+\frac{\sqrt{2}}{\pi}\left[\theta_{c}(x, \tau)-\theta_{c}(0,0)\right] \\
& =n x+\frac{1}{\pi}[\theta(x, \tau)-\theta(0,0)],
\end{aligned}
$$

where the spinless fields satisfy the same commutation relations as the original charge fields: $\left[\theta(x), \partial_{x^{\prime}} \phi\left(x^{\prime}\right)\right]$ $=\left[\theta_{c}(x), \partial_{x^{\prime}} \phi_{c}\left(x^{\prime}\right)\right]=i \pi \delta\left(x-x^{\prime}\right)$. We now turn to an evaluation of Eq. (13) for the cases of large and small $x$.

\section{1. $\mathcal{G}_{\sigma}(x, \tau)$ for large $x$}

When $x$ is large, $N(x, \tau)$ is also large and little error is made in converting the sum in Eq. (13) to an integral, $\Sigma_{m} \rightarrow \int d m$. When this is done, the integral over $m$ is readily evaluated with the delta function and one obtains

$$
\mathcal{G}_{\sigma}(x, \tau) \sim\left\langle(-1)^{N(x, \tau)}(2 S+1)^{-\mid N(x, \tau)} e^{i[\phi(x, \tau)-\phi(0,0)]}\right\rangle,
$$

where the expectation value over the charge degrees of freedom is taken in the limit $T \rightarrow 0 .{ }^{11}$ In order to deal with noninteger $N$, we write $(-1)^{N}=\operatorname{Re}\left[e^{i \pi N}\right]=\frac{e^{i \pi N}+e^{-i \pi N}}{2}$, the simplest form correct for integer $N$ (the harmonic approximation violates this) and consistent with the requirement that $\mathcal{G}_{\sigma}(x, \tau)$ be real and even in $x$. As a result, the Green's function takes the form $\mathcal{G}_{\sigma}(x, \tau)=\mathcal{G}_{\sigma,+}(x, \tau)$ $+\mathcal{G}_{\sigma,-}(x, \tau)$, with $\mathcal{G}_{\sigma,+}(x, \tau)=\left[\mathcal{G}_{\sigma,-}(x, \tau)\right]^{*}$. Making the definitions $\Phi(x, \tau) \equiv \phi(x, \tau)-\phi(0,0) \quad$ and $\quad \Theta(x, \tau) \equiv \theta(x, \tau)$

\footnotetext{
${ }^{11}$ All of the spin-incoherent results presented are computed at zero temperature in the charge sector. This is equivalent to taking the order of limits $J \rightarrow 0$, then $T \rightarrow 0$.
}

$-\theta(0,0)$, we use the Gaussian action resulting from Eq. (8) to move the averages to the exponent,

$$
\begin{aligned}
\mathcal{G}_{\sigma,+}(x, \tau) \sim & e^{-2 k_{F}|x| \ln (2 S+1) / \pi} e^{i 2 k_{F} x} \\
& \times\left\langle e^{i[1+i \ln (2 S+1) / \pi] \Theta(x, \tau)} e^{i \Phi(x, \tau)}\right\rangle \\
= & e^{-2 k_{F}|x| \ln (2 S+1) / \pi} e^{i 2 k_{F} x} e^{-(1 / 2)[1+i \ln (2 S+1) / \pi]^{2}\left\langle\Theta^{2}\right\rangle} \\
& \times e^{-(1 / 2)\left\langle\Phi^{2}\right\rangle} e^{-[1+i \ln (2 S+1) / \pi]\langle\Phi \Theta\rangle} .
\end{aligned}
$$

Standard computations from Eq. (8) using the relation (14) to relate $\theta=\sqrt{2} \theta_{c}$ and $\phi=\phi_{c} / \sqrt{2}$ give $\left\langle\Theta^{2}\right\rangle=K_{c} \ln \left(x^{2}\right.$ $\left.+v_{c}^{2} \tau^{2}\right), \quad\left\langle\Phi^{2}\right\rangle=\frac{1}{4 K_{c}} \ln \left(x^{2}+v_{c}^{2} \tau^{2}\right)$, and $\langle\Phi \Theta\rangle=\frac{1}{2} \ln \left(\frac{v_{c} \tau-i x}{v_{c} \tau+i x}\right)$. Substituting these values into Eq. (16), we find

$$
\begin{aligned}
& \mathcal{G}_{\sigma}(x, \tau)=\frac{C^{\prime} e^{-2 k_{F}|x| \ln (2 S+1) / \pi}}{\left(x^{2}+v_{c}^{2} \tau^{2}\right)^{\Delta_{K_{c}}}} \\
& \times\left(\frac{e^{i\left(2 k_{F} x-\varphi_{K_{c}}^{+}\right)}}{v_{c} \tau-i x}+\frac{e^{-i\left(2 k_{F} x-\varphi_{K_{c}}^{-}\right)}}{v_{c} \tau+i x}\right),
\end{aligned}
$$

where $C^{\prime}$ is an undetermined constant. ${ }^{12}$ The anomalous exponent $\Delta_{K_{c}}$ determining the power-law decay is given by

$$
\Delta_{K_{c}}=\frac{1}{8 K_{c}}+\frac{K_{c}}{2}\left[1-\left(\frac{\ln (2 S+1)}{\pi}\right)^{2}\right]-\frac{1}{2},
$$

and the additional phase factors coming from the

$$
\begin{aligned}
-\frac{\ln (2 S+1)}{\pi}\left(\left\langle\Theta^{2}\right\rangle+\langle\Phi \Theta\rangle\right)= & -\frac{\ln (2 S+1)}{\pi} \\
& \times\left[K_{c} \ln \left(x^{2}+v_{c}^{2} \tau^{2}\right)\right. \\
& \left.+\frac{1}{2} \ln \left(\frac{v_{c} \tau-i x}{v_{c} \tau+i x}\right)\right]
\end{aligned}
$$

piece in the exponent are

$$
\begin{aligned}
\varphi_{K_{c}}^{ \pm}(x, \tau)= & {[\ln (2 S+1) / \pi] } \\
& \times\left[K_{c} \ln \left(x^{2}+v_{c}^{2} \tau^{2}\right) \pm \frac{1}{2} \ln \left(\frac{v_{c} \tau-i x}{v_{c} \tau+i x}\right)\right] .
\end{aligned}
$$

For the special case of spin $S=1 / 2$ fermions (such as electrons) the result (17) reduces to Eq. (2) from the Introduction. In the case of infinite strength, zero range interactions [when $K_{c}=1 / 2$ (Schulz, 1990)] Eqs. (17)-(19) reduce to the tour-de-force Bethe-ansatz results of Cheianov and Zvonarev (2004a, 2004b).

There are several features of Eq. (17) worth emphasizing. First, note that the exponential decay [coming from the nonfluctuating part of $\left.(2 S+1)^{-|N|}\right]$ puts the correlations of the spin-incoherent Luttinger liquid out of the Luttinger liquid universality class because the correlation functions do not contain exclusively power-law

\footnotetext{
${ }^{12}$ Cheianov and Zvonarev (2004a) determined it for the special case of infinite strength zero range interactions.
} 
decays. ${ }^{13}$ Second, note that no parameter of the spin Hamiltonian (aside from the actual value of the spin itself) appears in the correlation function. This is an explicit example of the superuniversal spin physics in which all parameters (including the symmetry) of the spin Hamiltonian have dropped out. It is interesting that the actual value of the spin $S$ sets the scale of decay in both the exponential factor and the power-law piece (via $\left.\Delta_{K_{c}}\right)$. Aside from these differences, the spin-incoherent Luttinger liquid Green's function looks similar to that of the Luttinger liquid (1) insofar as it also possesses the right/left moving structure. However, as shown shortly, the tunneling density of states (which is derived from the single-particle Green's function) of the spin-incoherent Luttinger liquid is remarkably different from that of the Luttinger liquid. In order to investigate this in detail we need a more accurate form for $\mathcal{G}_{\sigma}(x, \tau)$ at small $x$. We turn to this now.

\section{2. $\mathcal{G}_{\sigma}(x, \tau)$ for small $x$}

Having discussed the spatial asymptotics of $\mathcal{G}_{\sigma}(x, \tau)$, for $|x| \rightarrow \infty$, we now turn our attention to the small $x$ limit which will allow us to compute the low-energy tunneling density of states at a point (when $x=0$ ). Unlike the situation with $x \rightarrow \infty$, when computing the Green's function at small $x$ one should be careful to take into account the discreteness of the number of world lines that may "bend" in between $(0,0)$ and $(x, \tau)$ (Fiete, Qian, et al., 2005):

$$
\begin{aligned}
\mathcal{G}_{\sigma}(x, \tau) \sim & \sum_{m=-\infty}^{\infty}(2 S+1)^{-|m|}(-1)^{m} \\
& \times\left\langle\delta(N(x, \tau)-m) e^{i[\phi(x, \tau)-\phi(x, 0)]}\right\rangle \\
= & \sum_{m=-\infty}^{\infty}(2 S+1)^{-|m|}(-1)^{m} \\
& \times \int \frac{d \lambda}{2 \pi} e^{-i \lambda m}\left\langle e^{i[\lambda \Theta(0, \tau) / \pi+\Phi(0, \tau)]}\right\rangle \\
\approx & \sqrt{\frac{\pi}{2\left\langle\Theta^{2}\right\rangle}} \sum_{m=-\infty}^{\infty}(2 S+1)^{-|m|}(-1)^{m} \\
& \times e^{-\pi^{2}(\bar{n} x-m)^{2} / 2\left\langle\Theta^{2}\right\rangle} e^{-\left\langle\Phi^{2}\right\rangle / 2} \\
= & \frac{a}{\sqrt{2 \pi} \bar{u}(\tau)}\left(\frac{a}{v_{c} \tau}\right)^{1 / 4 K_{c}} f(x, \bar{u}(\tau)),
\end{aligned}
$$

where

$$
f(z, \bar{u}) \equiv \sum_{m=-\infty}^{\infty} 2^{-|m|}(-1)^{m} e^{-(x-m a)^{2} / 2 \bar{u}^{2}}
$$

and $\bar{u}=\frac{a}{\pi} \sqrt{\left\langle\Theta^{2}\right\rangle}$. When $x=0$ we find (Fiete and Balents, 2004)

\footnotetext{
${ }^{13}$ I emphasize again that the limit $T \rightarrow 0$ has been taken, so this is an exponential decay at zero temperature.
}

$$
\begin{aligned}
\mathcal{G}_{\sigma}(0, \tau) \sim & \frac{1}{\sqrt{\tau^{1 / 2 K_{c}} \ln \left(v_{c} \tau\right)}} \sum_{m=-\infty}^{\infty}(2 S+1)^{-|m|}(-1)^{m} \\
& \times e^{-\pi^{2} m^{2} / 4 K_{c} \ln \left(v_{c} \tau\right)} \\
\sim & \frac{1}{\sqrt{\tau^{1 / 2 K_{c} \ln \left(v_{c} \tau\right)}}},
\end{aligned}
$$

where the final result (22) is obtained by noting that the sum over $k$ depends only weakly on $\tau$ and ranges between $S /(S+1)$ and 1 . This again recovers the Betheansatz results of Cheianov and Zvonarev (2004a, 2004b) for the case of infinite strength zero range interactions where $K_{c}=1 / 2$ (Schulz, 1990). We will see momentarily that Eq. (22) directly gives the tunneling density of states.

Before leaving the discussion of the single-particle Green's function, it is important to emphasize why the physics of the correlation functions are separated into those derived from particle nonconserving and particle conserving operators. The difference between these two types of operators (and their correlation functions) appears in the evaluation of the trace over the spin degrees of freedom in the spin-incoherent regime for which we used the noncrossing approximation to obtain a dominant contribution from trajectories that wrap around the imaginary time torus like those shown in Fig. 2(a). This class of trajectories is only possible for operators that create an "end point" of a world line, and these are precisely the operators that change particle number. These trajectories are responsible for the $(2 S+1)^{-|N(x, \tau)|}$ factors and all associated spin-incoherent effects such as the exponential decay with distance of the single-particle Green's function (17) and the logarithmic time dependence in Eq. (22). For a particle number conserving operator it is not possible to simultaneously satisfy the noncrossing approximation and have wraparound trajectories. In fact, in Sec. IV we show that for quantities derived from particle conserving operators there is a precise mapping in the spin-incoherent regime between a spin-incoherent Luttinger liquid and a spinless Luttinger liquid. We have already seen hints of this in the spinless topology of the world line configurations after tracing over the spin degrees of freedom and in the operator relations between $\theta$ and $\theta_{c}$ in Eq. (14).

A related issue to when it is possible to have wraparound trajectories is what happens to the form of the Green's function in the spin-incoherent regime when a particle is added near the boundary of the system, such as at the end of a semi-infinite wire. At the end of a wire, the density fluctuations are effectively frozen out so that $N(0, \tau) \equiv 0$. From the first line of Eq. (20) this implies that only the $m=0$ term in the sum can contribute,

$$
\mathcal{G}_{\sigma, \text { end }}(0, \tau) \sim\left\langle e^{i[\phi(0, \tau)-\phi(0,0)]}\right\rangle_{\mathrm{end}} \sim \frac{1}{\tau^{1 / 2 K_{c}}},
$$

which compared to Eq. (22) has a doubled exponent and the logarithmic correction characteristic of the spin incoherence is absent. Up to a factor of 2 in the exponent, 
Eq. (23) is identical to the result that one would obtain for a spinless Luttinger liquid. It is our second hint that the spin-incoherent Luttinger liquid may be related to a spinless Luttinger liquid in some ways. Results for the Green's function near, but not precisely at, the end of a boundary have been discussed by Fiete, Qian, et al. (2005), Kakashvili and Johannesson (2006a), and Kindermann and Brouwer (2006).

\section{Tunneling density of states}

One of the most remarkable differences between a spin-incoherent Luttinger liquid and a Luttinger liquid is the frequency (energy) dependence of the tunneling density of states. It is well known (Glazman et al., 1992; Voit, 1995; Gogolin et al., 1998; Giamarchi, 2004) that the tunneling density of states in a Luttinger liquid is suppressed as a power law (3) at low energies due to orthogonality catastrophe-type effects resulting from the interactions: when a new particle is added to the system the others must rearrange themselves to accommodate the additional particle. The resulting final-state wave function is orthogonal to the original one in the limit of large particle numbers. While the physics of the orthogonality catastrophe is still operational in the spinincoherent Luttinger liquid, the large number of highly excited spin states at low energy turns out to dramatically affect the tunneling density of states.

The tunneling density of states is obtained (Cheianov and Zvonarev, 2004a, 2004b; Fiete and Balents, 2004) by Fourier transforming Eq. (22),

$$
A(\omega) \sim \omega^{\left(1 / 4 K_{c}\right)-1 / \sqrt{|\ln (\omega)|}, \quad \hbar \omega \geqslant k_{B} T .}
$$

Note that while the exponent is positive for any value of $K_{c}$ in Eq. (3), for the spin-incoherent case the exponent is only positive if $K_{c}<1 / 4$. The value of $K_{c}$ for the infinite $U$ limit of the Hubbard model is $1 / 2$ (Schulz, 1990) and to obtain a smaller $K_{c}$ one needs a combination of both very strong and longer ranged interactions. As a result, many systems will likely have $K_{c}>1 / 4$ and the tunneling density of states will exhibit a divergence at low energies for $\hbar \omega \gtrsim k_{B} T$. This is qualitatively distinct from the Luttinger liquid and should be a relatively simple feature to observe in experiment. The divergence in Eq. (24) has a straightforward interpretation: Orthogonality catastrophe-type physics operational in the charge sector competes with a highly degenerate spin sector at very low energies. For $K_{c}>1 / 4$ the huge availability of spin states at low energies "beats" the orthogonality catastrophe and leads to an apparent divergence for $\hbar \omega \geqslant k_{B} T$, while for $K_{c}<1 / 4$, the interactions in the charge sector are sufficiently strong to create an orthogonality catastrophe that overwhelms the degenerate spin states and a power-law suppression of the tunneling density of states is recovered.

The result (24) has been refined and extended to negative frequencies by Matveev et al. (2007). In the spinincoherent regime, the tunneling density of states possesses an asymmetry $A(\omega)=2 A(-\omega)$. The behavior is shown schematically in Fig. 3.

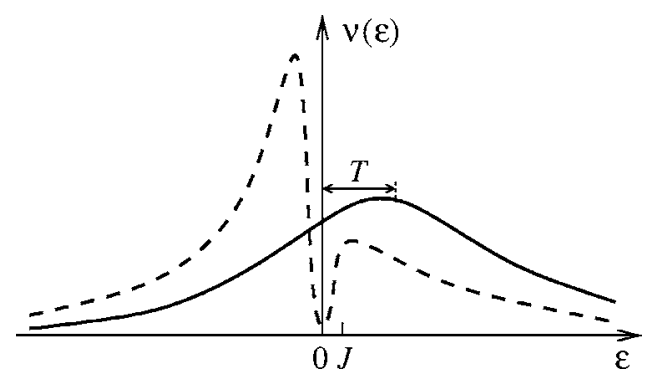

FIG. 3. Schematic of the tunneling density of states $\nu(\varepsilon)$ $\equiv A(\omega)$. The solid (dashed) line is the high- (low-) temperature regime $k_{B} T \gg J\left(k_{B} T \ll J\right)$. In the high-temperature regime when $\varepsilon \gg k_{B} T, \nu(\varepsilon)=2 \nu(-\varepsilon)$. When the temperature is lowered below the spin energy $J$, the tunneling density of states at $\varepsilon$ $<0$ grows by roughly a factor of 3 , while for $\varepsilon>0$ it decreases dramatically. For $k_{B} T \ll \varepsilon$ and $\varepsilon \ll J$ the standard Luttinger liquid behavior of the power-law suppression is observed at small $\varepsilon$. From Matveev et al., 2007.

\section{Finite magnetic field}

The spin-incoherent Luttinger liquid can be studied in a straightforward way in the presence of an external magnetic field. It is assumed for this part of the discussion that there are no significant orbital effects from the external field. This is certainly true if the system is strictly one dimensional, but real systems such as quantum wires are quasi-one-dimensional and orbital effects may be important for sufficiently large fields. Neglecting any orbital coupling, the external field $B$ only couples to the spin of the particles (assumed $S=1 / 2$ here for simplicity),

$$
H_{Z}=-g_{e} \mu_{B} B \sum_{l} S_{l}^{z},
$$

where $g_{e}$ is the gyromagnetic ratio of the electron and $\mu_{B}$ is the Bohr magneton.

In the absence of a magnetic field, the Green's functions satisfy $\mathcal{G}_{\uparrow}(x, \tau)=\mathcal{G}_{\downarrow}(x, \tau)$. But this is no longer the case in the presence of a magnetic field. Nevertheless, all of the arguments used in the beginning of Sec. III.A to evaluate the trace over the spin degrees of freedom using the noncrossing approximation still hold as they are based only on $E_{\text {spin }} \ll k_{B} T$. The only change is that now the probability of having spin projection $\sigma$ along the $z$ axis depends on $\sigma$ and the value of the external field. In particular, the factor $(2 S+1)^{-|m|}$ in Eq. (13) gets modified to ${ }^{14} p_{\sigma}^{|m|+1}$ with $p_{\uparrow}=\left(1+\exp \left\{-E_{Z} / k_{B} T\right\}\right)^{-1}$ and $p_{\downarrow}=1-p_{\uparrow}$, where $E_{Z}=2 g_{e} \mu_{B} B$ is the Zeeman energy of an electron in a magnetic field referenced to the minimum-energy configuration with the spin parallel to the field.

\footnotetext{
${ }^{14}$ We have actually dropped an unimportant overall factor of $(2 S+1)^{-1}$ in Eq. (13), but in discussing finite magnetic fields we must include this additional factor in the form of its finite field generalization $p_{\sigma}$. The probability of having $N$ spins aligned is $(2 S+1)^{-|N|}$, but the probability of having $N$ spins aligned in a particular direction is $(2 S+1)^{-1} \times(2 S+1)^{-|N|}$, giving the extra multiplicative factor.
} 

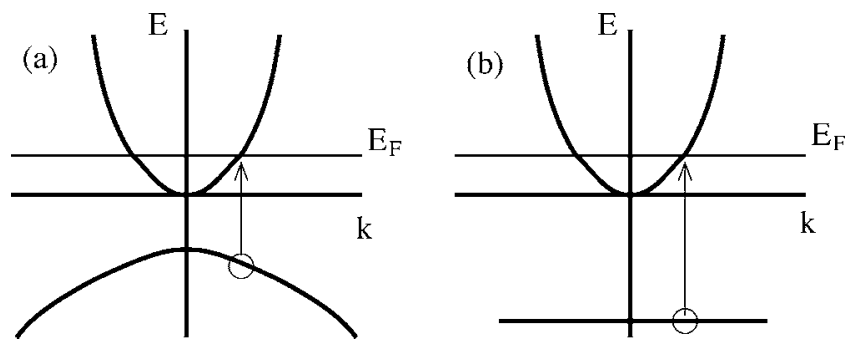

FIG. 4. Schematic of threshold photoexcitation for (a) finite hole mass and (b) infinite hole mass. (a) An electron is excited from the valence band to the conduction band leaving behind a hole in valence band. (b) An electron is excited from a deep (infinite mass) core level. We assume the conduction band is occupied by a spin-incoherent Luttinger liquid.

All calculations presented so far carry through as before only with the changes above. Kindermann and Brouwer (2006a) have studied these effects on the single-particle Green's function and the tunneling density of states. ${ }^{15}$ The main result is that a new time scale,

$$
\tau_{B}=\frac{a}{v_{c}} e^{E_{Z} / K_{c} k_{B} T}
$$

is introduced which sets a cutoff for spin-incoherent effects. For times much longer than this spin-incoherent Luttinger liquid effects are observed, while for times much shorter than this, the system behaves like a spinless Luttinger liquid. In the limit of very large external fields, $E_{Z} \rightarrow \infty$, and therefore $\tau_{B} \rightarrow \infty$, there is no time (frequency) range over which spin-incoherent Luttinger liquid physics may be observed. This result, of course, squares with intuition: A fully polarized spin-incoherent Luttinger liquid should be identical to a spinless Luttinger liquid. This is indeed the case.

\section{B. Fermi-edge singularity}

In the Fermi-edge singularity, an incoming photon excites a deep "core" level electron up to the Fermi energy as shown schematically in Fig. 4. There is a minimum energy required to do this ${ }^{16}$ called the threshold energy and the energy dependence of the photon absorption just above the threshold energy is what constitutes the Fermi-edge singularity, as it is often singular. The detailed form of the singularity can reveal a great deal about the interactions in the system, the mass of the core hole created, and the absence or presence of spinincoherent degrees of freedom (Fiete, 2006).

The photon absorption rate is computed by Fermi's golden rule and is given by

\footnotetext{
${ }^{15}$ External magnetic-field effects on the Fermi-edge singularity (Fiete, 2006) and the momentum structure in momentum resolved tunneling (Fiete, Qian, et al., 2005) have also been studied.

${ }^{16}$ In two and three dimensions, this statement is only strictly true if the core level is infinitely massive.
}

$$
I(\omega) \propto \sum_{\sigma} \operatorname{Re} \int_{0}^{\infty} d t e^{i \omega t}\left\langle\psi_{\sigma}(t) h_{\sigma}(t) h_{\sigma}^{\dagger}(0) \psi_{\sigma}^{\dagger}(0)\right\rangle,
$$

where as before the operator $\psi_{\sigma}^{\dagger}\left(\psi_{\sigma}\right)$ creates (annihilates) an electron and $h_{\sigma}^{\dagger}\left(h_{\sigma}\right)$ creates (annihilates) a hole with spin $\sigma$. Equation (27) shows that the photon absorption is related to a correlation function derived from particle nonconserving operators $h_{\sigma}^{\dagger} \psi_{\sigma}^{\dagger}$ and its Hermitian conjugate. We therefore expect spin-incoherent effects to be manifest.

The result of an analysis by Fiete (2006) is that the Fermi-edge singularity falls into two classes of behavior depending on whether the hole created by the photon is localized (infinitely massive) or delocalized (finite mass). The important distinction between the two cases is that the former breaks the translational symmetry of the system while the latter does not. The most significant consequence of the breaking of translational symmetry is that backscattering from an infinitely massive impurity is relevant (in a system with repulsive interactions) and cuts the electron system into two semi-infinite parts (Kane and Fisher, 1992; Furusaki and Nagaosa, 1993), while backscattering from a finite mass impurity is irrelevant (Castro Neto and Fisher, 1996). These two limits have important implications for the boundary conditions that the $\theta$ and $\phi$ operators appearing in $\psi_{\sigma}$ satisfy. ${ }^{17}$

In the finite hole case shown in Fig. 4(a) one can transform to a frame comoving with the excited hole (Castro Neto and Fisher, 1996; Tsukamoto, 1998a, 1998b). In this frame the Hamiltonian takes the form $H=H_{\text {elec }}$ $+H_{\text {elec-hole }}+H_{\text {hole }}$, where

$$
H_{\text {elec-hole }}=\frac{U_{s}^{f}}{\pi} h^{\dagger} h \partial_{x} \theta(0) \pm \frac{U_{a}^{f}}{\pi} h^{\dagger} h \partial_{x} \phi(0),
$$

$H_{\text {elec }}=H_{c}+H_{s}, \quad H_{\text {hole }}=\Sigma_{\sigma} E_{h, \sigma} h_{\sigma}^{\dagger} h_{\sigma}, \quad$ and $\quad h^{\dagger} h=\Sigma_{\sigma} h_{\sigma}^{\dagger} h_{\sigma}$. Here $U_{s}^{f}$ is the symmetric part of forward scattering from the hole and $U_{a}^{f}$ is the antisymmetric part of the forward scattering (Tsukamoto et al., 1998a). (In our convention $\partial_{x} \theta$ represents the density fluctuations and $\partial_{x} \phi$ is the particle current.) The antisymmetric part appears since in the frame of the hole, it sees a net current of particles scattering from it. The + sign is for a right-moving hole and the - sign is for a left-moving hole. The parameter $U_{a}^{f}$ depends on the momentum and mass of the hole, and when it is at rest, $U_{a}^{f} \equiv 0$ (Tsukamoto et al., 1998a). Since backscattering from a finite mass impurity is not relevant it has no effect on the Fermi-edge physics and therefore has not been included in $H_{\text {elec-hole. The }}$ Hamiltonian $H$ can be diagonalized with the unitary transformation $U=\exp \left\{-i\left[\delta_{a} \theta(0)+\delta_{s} \phi(0)\right] h^{\dagger} h\right\} \quad$ where $\delta_{a} \equiv \mp U_{a}^{f} / v_{c} K_{c} \pi$ and $\delta_{s} \equiv-2 K_{c} U_{s}^{f} / v_{c} \pi$. Applying this

\footnotetext{
${ }^{17}$ Recall that the Green's function for tunneling into a point (22) in an infinite system is different from the Green's function for tunneling into the end (23) of a semi-infinite system because of the different boundary conditions on $\theta$. As before, the spinless $\theta$ and $\phi$ fields are related to the spinful ones via Eq. (14) and just below it.
} 
transformation we find $\bar{H} \equiv U^{\dagger} H U=H_{\text {elec }}+\bar{H}_{\text {hole }}$, where the only change to $H_{\text {hole }}$ is a shift in the hole energy $E_{h, \sigma} \rightarrow \tilde{E}_{h, \sigma^{*}}$. The correlation function appearing in Eq. (27) can now be computed in imaginary time,

$$
\begin{aligned}
C_{\sigma}(\tau) & =\frac{1}{Z} \operatorname{Tr}\left[e^{-\beta H} \psi_{\sigma}(\tau) h_{\sigma}(\tau) h_{\sigma}^{\dagger}(0) \psi_{\sigma}^{\dagger}(0)\right] \\
& =\frac{1}{Z} \operatorname{Tr}\left[e^{-\beta \bar{H}} \bar{\psi}_{\sigma}(\tau) \bar{h}_{\sigma}(\tau) \bar{h}_{\sigma}^{\dagger}(0) \bar{\psi}_{\sigma}^{\dagger}(0)\right],
\end{aligned}
$$

where in the second line $U U^{\dagger}=1$ has been inserted and the cyclic property of the trace has been exploited. Direct evaluation gives $\bar{\psi}_{\sigma}=\psi_{\sigma}$ (up to unimportant multiplicative factors) and $\bar{h}_{\sigma}=h_{\sigma} e^{-i\left[\delta_{a} \theta+\delta_{s} \phi\right]}$, so that

$$
\begin{aligned}
C_{\sigma}(\tau)= & \frac{e^{-\tilde{E}_{h, \sigma} \tau}}{Z_{\text {elec }}} \operatorname{Tr}\left[e^{-\beta H_{\text {elec }}} \psi_{\sigma}(\tau) e^{-i\left[\delta_{a} \theta(\tau)+\delta_{s} \phi(\tau)\right]}\right. \\
& \left.\times e^{i\left[\delta_{a} \theta(0)+\delta_{s} \phi(0)\right]} \psi_{\sigma}^{\dagger}(0)\right],
\end{aligned}
$$

which is now of exactly the same form as the Green's function (12). Carrying through the same manipulations outlined in Sec. III.A, we find

$$
I(\omega) \propto \frac{\left(\omega-\omega_{\mathrm{th}}\right)^{\left(1 / 4 K_{c}\right)\left(1-\delta_{s}\right)^{2}-1}}{\sqrt{\left|\ln \left(\omega-\omega_{\mathrm{th}}\right)\right|} \Theta\left(\omega-\omega_{\mathrm{th}}\right)},
$$

where $\Theta\left(\omega-\omega_{\mathrm{th}}\right)$ is the step function and $\omega_{\mathrm{th}}=\tilde{E}_{h, \sigma} / \hbar$ is the threshold frequency. A few of the most important features of Eq. (30) are worth emphasizing. First, in contrast to the spin-coherent (spin-polarized) Luttinger liquid (Tsukamoto et al., 1998a, 1998b) the threshold exponent does not depend on the mass of the core hole. Second, there are universal (independent of interactions) logarithmic corrections to the power-law threshold behavior. These logarithmic corrections are of the same nature as those that arose in the tunneling density of states in the spin-incoherent Luttinger liquid, Eq. (24). Third, there is indeed a minimum (threshold) frequency for absorption.

The case of infinite hole mass shown in Fig. 4(b) is qualitatively different because now the hole is a relevant perturbation. At frequencies just above the threshold frequency $\omega_{\text {th }}$, the hole acts just as if it were an "end" in the system (Kane and Fisher, 1992; Furusaki and Nagaosa, 1993). As a result, the effective low-energy boundary condition is $\theta(\tau)=0$, just as we found for the Green's function at the end of a semi-infinite system, Eq. (23). The Fermi-edge singularity therefore maps onto an equivalent spinless problem. It has been shown that for a spinless Luttinger liquid the infinitely massive core hole leads to a universal backscattering contribution to the exponent of 1/8 (Gogolin, 1993; Prokof'ev, 1994; Furusaki, 1997; Kominik et al., 1997) and this leads to the infinite mass threshold result $I(\omega) \propto(\omega$ $\left.-\omega_{\text {th }}\right)^{\left(1 / 2 K_{c}\right)\left(1-\delta_{S}\right)^{2}+1 / 8-1} \Theta\left(\omega-\omega_{\text {th }}\right)$. As with the Green's function at the end of a semi-infinite system, there is no logarithmic correction to the frequency dependence. For symmetry reasons mentioned before there is nonanalytical behavior in the exponent when the infinite mass limit is taken for the hole (Castella, 1996).

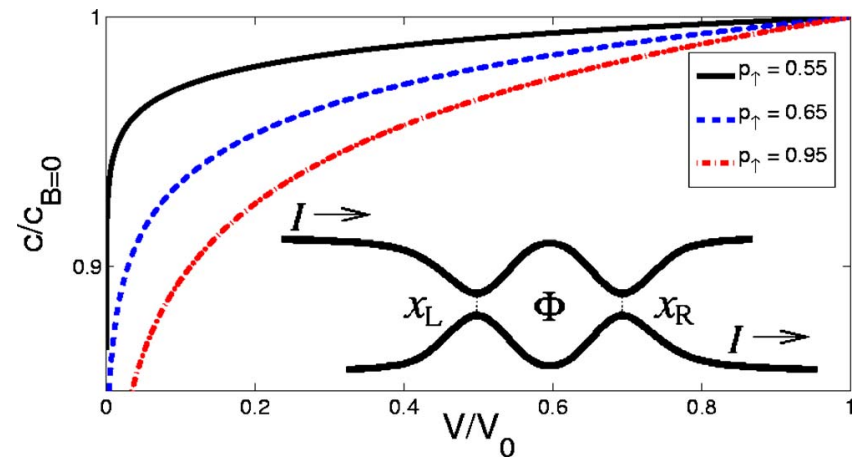

FIG. 5. (Color online) The interference contrast (31) obeys a power law with a temperature- and magnetic-field-dependent exponent $2 K_{c}\left(1-\ln ^{2} p_{\uparrow} / \pi^{2}\right)$, Eq. (32). (Interference schematic in the inset.) Plotted is $c=C(V) / C\left(V_{0}\right)$, the interference contrast normalized with respect to an arbitrary reference voltage $V_{0}$. It is compared to its value at zero magnetic field $c_{B=0}$ for various polarizations $p_{\uparrow}$ with $K_{c}=1 / 2$. From Kindermann et al., 2006.

\section{Spin-incoherent effects in transport}

Transport experiments in mesoscopics are by now fairly routine and provide fertile ground for observing spin-incoherent effects. In this subsection we summarize some of the results from the theory of the spinincoherent Luttinger liquid that can be readily probed in transport experiments.

Spin-incoherent effects are expected to have dramatic consequences on interference experiments such as those shown schematically in the inset of Fig. 5. This situation has been studied in detail by Kindermann et al. (2006) and Le Hur (2006). In this type of interference experiment two quantum wires are in close proximity and tunneling dominates at two spatial points, $x_{L}$ and $x_{R}$. A flux $\Phi$ penetrates the region between these wires bounded by $x_{L}$ and $x_{R}$ as indicated in the figure. A current $I$ is injected in the top wire to the left of $x_{L}$ and the resulting current is measured in the lower wire to the right of $x_{R}$. For the current in the lower wire to be finite, electrons must tunnel from the upper wire at either $x_{L}$ or $x_{R}$. In general, there will be a nonzero amplitude for each and depending on the flux $\Phi$ there will be either constructive or destructive interference (provided the two paths are coherent) leading to an oscillating current as a function of $\Phi$.

A convenient way to quantify the coherence of the system is with the interference contrast $C$,

$$
C=\frac{\sqrt{\left\langle\left[I(\varphi)-\langle I\rangle_{\varphi}\right]^{2}\right\rangle}}{\langle I\rangle_{\varphi}}, \quad\langle\cdots\rangle_{\varphi}=\int_{0}^{2 \pi} \frac{d \varphi}{2 \pi} .
$$

Kindermann et al. (2006) showed that in the spinincoherent regime the contrast behaves as

$$
C \sim p_{\uparrow}(e V)^{2 K_{c}\left(1-\ln ^{2} p_{\uparrow} / \pi^{2}\right)},
$$

where $p_{\uparrow}=\left(1+e^{-E_{Z} / k_{B} T}\right)^{-1}$ is the probability of having a spin-up electron. The interference contrast exhibits an 


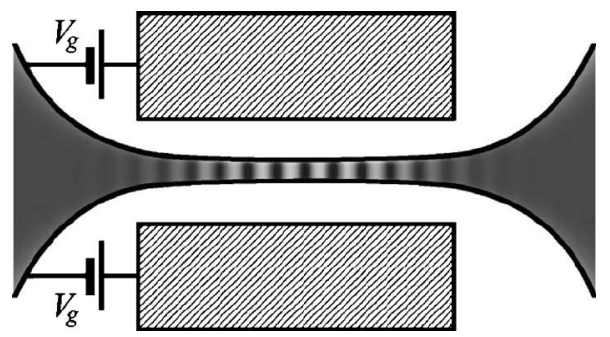

FIG. 6. Schematic of a quantum wire attached to Fermi-liquid leads. The electron density is assumed to be small near the center of the wire so that $r_{s}$ is large and strong Wigner solid correlations are present. In the leads to the left and right of the wire the electrons are assumed to be noninteracting. From Matveev, 2004b.

anomalous scaling with voltage, magnetic field, and temperature. Numerical results are presented in Fig. 5.

So far, we have dealt only with topics that involve infinite or semi-infinite systems. In any real transport situation a quasi-one-dimensional system will ultimately be connected to Fermi-liquid leads and this will affect many aspects of transport. For example, the dc conductance of an infinite single mode spinless Luttinger liquid with interaction parameter $g$ is $g \frac{e^{2}}{h}$ (Kane and Fisher, 1992), while if Fermi liquid leads are attached the dc conductance becomes $\frac{e^{2}}{h}$ independent of $g$ (Maslov and Stone, 1995; Safi and Schulz, 1995). It is therefore important to know how the finite length of the wire and the Fermi-liquid leads will affect the observation of spinincoherent Luttinger liquid physics.

Pioneering work in this direction was done by Matveev $(2004 \mathrm{a}, 2004 \mathrm{~b})$ in the context of the $0.7\left(2 e^{2} / h\right)$ conductance feature (Thomas et al., 1996; Cronenwett et al., 2002; DiCarlo et al., 2006) in quantum point contacts. Matveev modeled the quantum point contact as a finite length quantum wire of very low particle density adiabatically connected to Fermi-liquid leads, as shown in Fig. 6. The conductance was computed by using a Wigner solid model like that given in Eqs. (7) and (8) where $J_{l}$ was assumed to be spatially dependent. For electrons near the center of the wire $J_{l} \ll E_{F}$, while it grew to of order $E_{F}$ in the leads. Remarkably, when the temperature of the system is such that the wire is in the spin-incoherent regime, $J \ll k_{B} T \ll E_{F}$, the dc conductance of the wire is reduced to half the noninteracting value, $0.5\left(2 e^{2} / h\right)$, which is close to the $0.7\left(2 e^{2} / h\right)$ feature. One physical interpretation of this result is that when an electron in the leads with energy $\sim k_{B} T$ enters the constricted region of the wire it starts to decompose into separate spin and charge components. Since the bandwidth of the spin modes scales as $J_{l} \ll k_{B} T$ there are no propagating spin modes in the wire at the energy of the electron and these states are all reflected while the charge modes are allowed to pass through. This argument explains the reduction of the conductance. The factor of 2 reduction (relative to the noninteracting value of $2 e^{2} / h$ ) is more subtle and appears to depend on the symmetry of the spin Hamiltonian in the wire

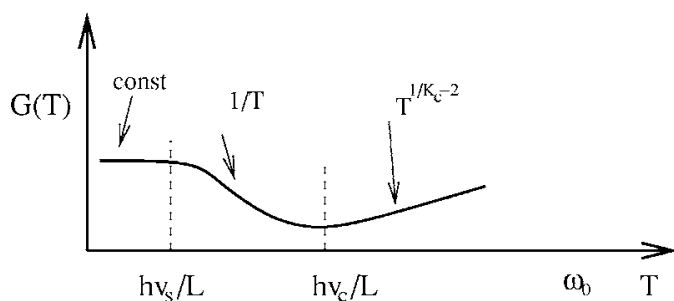

FIG. 7. Schematic of the temperature dependence of a quantum wire of length $L$ attached to Fermi-liquid leads with a strong impurity in the center. For a range of temperatures $\hbar v_{s} / L \ll k_{B} T \ll \hbar v_{s} / L$ the charge modes have propagated into the leads, but the spin modes have not, leading to a universal temperature dependence of the conductance in this energy window.

(Matveev, 2004a, 2004b). Recently this issue has also been addressed numerically (Syljuasen, 2007). Interestingly, in the regime $J \ll k_{B} T \ll E_{F}$ it appears the shot noise can also be dramatically reduced in certain situations (Kindermann and Brouwer, 2006b).

If a strong impurity is added to the center of a wire like that shown in Fig. 6, interesting nonmonotonic behavior of the conductance $G(T)$ may result (Fiete, Le Hur, and Balents, 2005). For a strong impurity, the conductance can be calculated using Eq. (23) with Fermi's golden rule for tunneling across the impurity. The result is schematically shown in Fig. 7. For $k_{B} T$ $\gg \hbar v_{c} / L, \hbar v_{s} / L$ the quantum wire behaves just like an infinite system because the excitations do not see the leads. When $\hbar v_{s} / L \ll k_{B} T \ll \hbar v_{c} / L$ the charge modes see the leads but the spin modes do not. Naively taking the noninteracting value of $K_{c}=1$ leads to an inverse temperature dependence down to temperatures $k_{B} T$ $\lesssim \hbar v_{s} / L$ where the system looks like a strong impurity in a Fermi liquid and the value of the conductance saturates.

\section{PARTICLE CONSERVING OPERATORS}

In some sense, the greatest qualitative differences between a spin-incoherent Luttinger liquid and a Luttinger liquid are seen in the correlation functions derived from the particle nonconserving operators that have been discussed up to this point. This is because of the particular class of trajectories possible [see Fig. 2(a)] for particle nonconserving operators in the noncrossing approximation. Upon evaluating the trace over the spin degrees of freedom, factors such as $(2 S+1)^{-|N|}$ (and its finite magnetic-field generalization $p_{\sigma}$ ) appear, leading to exponential decays in space (17) and logarithmic corrections in time (22). As mentioned before, such trajectories are not possible for particle conserving operators. Nevertheless, there are important ways in which spinincoherent effects manifest themselves for this class of operators, namely, in the temperature dependence of the correlation functions.

For this section the focus will be on only one particle conserving operator-the density. All of the physics dis- 
cussed here will be related to the weak $2 k_{F}$ density modulations in the large $r_{s}$, strongly interacting limit. For $k_{B} T \ll E_{\text {spin }}$ these $2 k_{F}$ density modulations will be present due to the weak spin-charge coupling, while for $k_{B} T \gg E_{\text {spin }}$ they will be thermally washed out. Therefore quantities depending on the $2 k_{F}$ density modulations, such as the Coulomb drag between parallel quantum wires and certain types of noise, will exhibit a sharp temperature dependence around $k_{B} T \approx E_{\text {spin }}$. Aside from the physics summarized in Fig. 7 in which a spin energy $\left(\hbar v_{s} / L\right)$ explicitly appeared, up to this point all of the physics discussed has explicitly or implicity taken $E_{\text {spin }}$ $\rightarrow 0$. In this section, it will be important to keep $E_{\text {spin }}$ finite in order to compute the $2 k_{F}$ density modulations. However, before we discuss that in detail, one more result is in order for the $E_{\text {spin }} \rightarrow 0$ limit. We turn to this now.

\section{A. Mapping to spinless electrons in the spin-incoherent Luttinger liquid}

In this subsection we show explicitly that in the spinincoherent Luttinger liquid electrons become effectively spinless (for quantities that do not directly probe spin or change particle number) and are governed by a Hamiltonian of the form (8) with interaction parameter $g=2 K_{c}$ (Fiete, Le Hur, and Balents, 2005).

To show this microscopically, we work in the canonical ensemble, i.e., with a fixed number of electrons. The dynamics in the grand canonical ensemble can be obtained from this by summing over the sectors with each electron number. For a fixed electron number, a convenient real-space basis set is given by states specifying the position $x_{l}$ of each electron, and the spin projection on the $\hat{z}$ axis, $\sigma_{l}$, in order, from left to right across the system:

$$
\left|x_{1} \cdots x_{N}\right\rangle\left|\sigma_{1} \cdots \sigma_{N}\right\rangle=c_{\sigma_{1}}^{\dagger}\left(x_{1}\right) \cdots c_{\sigma_{N}}^{\dagger}\left(x_{N}\right)|0\rangle
$$

where $|0\rangle$ is the vacuum state (no particles).

As we discussed in the context of the evaluation of the single-particle Green's function, the physics of the spinincoherent regime is that, within the thermal coherence time $t_{\text {coh }} \sim \hbar / k_{B} T$, the probability of a transition between states with different values of $\left\{\sigma_{n}\right\}$ is negligible. Hence the physics is well approximated by neglecting offdiagonal matrix elements in these states. Moreover, in the same approximation, for spin-independent interactions the matrix elements of $H$ are independent of $\left\{\sigma_{n}\right\}$,

$$
\begin{aligned}
& \left\langle\sigma_{1}^{\prime} \cdots \sigma_{N}^{\prime}\left|\left\langle x_{1}^{\prime} \cdots x_{N}^{\prime}|H| x_{1} \cdots x_{N}\right\rangle\right| \sigma_{1} \cdots \sigma_{N}\right\rangle \\
& \quad \approx\left\langle x_{1}^{\prime} \cdots x_{N}^{\prime}\left|H_{\text {spinless }}\right| x_{1} \cdots x_{N}\right\rangle \delta_{\sigma_{1}^{\prime}, \sigma_{1}} \cdots \delta_{\sigma_{N}^{\prime}, \sigma_{N}},
\end{aligned}
$$

where $H_{\text {spinless }}$ is an effective spinless Hamiltonian that governs the (independent) dynamics within each spin sector which must have the same form as Eq. (8). It only remains to determine the mapping between the coupling constants of $H_{c}$ and $H_{\text {spinless. }}$. To do this we equate a physical quantity between the two representations, such as the density. In fact, we have already done this in Eq. (14) which shows $\theta=\sqrt{2} \theta_{c}$ and $\phi=\phi_{c} / \sqrt{2}$. Upon substitu- tion into Eq. (8), this gives an identical collective mode velocity but a new coupling constant $g=2 K_{c}$ (Fiete, Le Hur, and Balents, 2005). This equivalence is completely general and continues to hold in the presence of arbitrary potentials, weak links, etc., so long as $J \ll k_{B} T$ throughout the system, there are no explicit spindependent interactions in the Hamiltonian, and electrons are not added or removed from the system during the dynamics. The single-particle Green's function for the infinite system (17) does not satisfy that last condition and that is why it does not map onto the Green's function of some spinless Luttinger liquid.

\section{B. Spin-charge coupling in Wigner solid model}

In the limit of large $r_{s}$, the dominant finite wavevector spatial correlations are at $4 k_{F}$ corresponding to an average spacing $a$ between particles, as schematically shown in Fig. 1. Recall that for a noninteracting electron gas the dominant finite wave-vector spatial correlations are at $2 k_{F}$ where $k_{F} \equiv \pi /(2 a)$. The difference between the two is simply understood because for noninteracting electrons two particles (a spin up and a spin down) can occupy the same position in space, while for large $r_{s}$ the interactions are so strong that two particles tend not to be near each other in space regardless of their relative spin orientation.

Returning for the moment to $T=0$, one may ask how the Wigner solid "crosses over" to the noninteracting limit in one dimension as a function of $r_{s} .{ }^{18}$ Starting from the fluctuation Wigner solid model (9) one can address this question by allowing coupling of the spin and charge degrees of freedom. In particular, the $2 k_{F}$ oscillations (characteristic of the weakly interacting case) can be seen to enter through the $l$ dependence of the coupling $J_{l}$ between spins in Eq. (7). Assuming that the local fluctuations from the equilibrium positions $u_{l}$ are small compared to the mean particle spacing $a$, the exchange energy can be expanded as (Fiete et al., 2006)

$$
J_{l}=J+J_{1}\left(u_{l+1}-u_{l}\right)+\mathcal{O}\left(\left(u_{l+1}-u_{l}\right)^{2}\right) .
$$

In this case the full Hamiltonian takes the form

$$
H=H_{c}+H_{s}\left(J_{l}=J\right)+H_{s-c},
$$

where

$$
H_{s-c}=J_{1} \sum_{l}\left(u_{l+1}-u_{l}\right) \vec{S}_{l+1} \cdot \vec{S}_{l} .
$$

Equation (36) explicitly couples the spin modes to the elastic distortions of the lattice constituting the charge

\footnotetext{
${ }^{18}$ Because of strong quantum fluctuations in one dimension, a true Wigner solid does not exist. However, the longer range the interactions, the more slowly the density correlations decay. It turns out that for Coulomb interactions, the correlations decay slower than any power law (Schulz, 1993).
} 
modes. The displacement $u_{l}$ of the $l$ th electron in the harmonic chain (5) and (36) can be expanded as

$$
u_{l}=u_{0}(l a)+u_{\pi}(l a)(-1)^{l},
$$

where $u_{0}$ refers to the $k \approx 0$ component of the displacement and $u_{\pi}$ refers to the $k \approx \pi / a \equiv 2 k_{F}$ displacement. Both $u_{0}$ and $u_{\pi}$ are assumed to be slowly varying functions of position, and we expect $u_{\pi} \ll u_{0}$ when the interactions are strong. When Eq. (37) is substituted into Eq. (36) and the continuum limit is taken, it is the $u_{\pi}\left(2 k_{F}\right)$ piece that will couple to the spin degrees of freedom. Since the spin sector is expected to have antiferromagnetic correlations (which therefore oscillate at wave vector $\left.2 k_{F}\right)$, this is the crucial coupling that will allow the $2 k_{F}$ correlations of the spin sector to "spill over" into the charge sector. Of course, there are only correlations in the spin sector provided $k_{B} T \ll E_{\text {spin. }}$. In this way the presence or absence of $2 k_{F}$ correlations in the charge density of a strongly interacting $1 \mathrm{D}$ system can tell about the relative size of $k_{B} T$ and $E_{\text {spin }}$.

\section{Description of the effective density}

This idea can by quantified by starting with the density representation $\rho(x, t)=\Sigma_{l} \delta\left(x-a l-u_{l}(t)\right)$. The density can be expanded in the displacements $u_{l}$, and the higher energy $u_{\pi}$ pieces can be integrated out to yield an effective density valid when $k_{B} T \ll E_{\text {spin }}$ (Fiete et al., 2006),

$$
\begin{aligned}
\rho^{\mathrm{eff}}(x, \tau)= & \rho_{0}-\frac{\sqrt{2}}{\pi} \partial_{x} \theta_{c}(x, \tau)-\frac{\rho_{0}}{16 \pi}\left(\frac{J_{1}}{m \omega_{0}^{2} a^{2}}\right) \\
& \times \sin \left[2 k_{F} x+\sqrt{2} \theta_{c}(x, \tau)\right] \sin \left[\sqrt{2} \theta_{s}(x, \tau)\right] \\
& +\rho_{0} \cos \left[4 k_{F} x+\sqrt{8} \theta_{c}(x, \tau)\right],
\end{aligned}
$$

where $\rho_{0}=1 / a$.

The density expression (38) has effectively traded the high-energy (because they are at the zone boundary) $u_{\pi}$ modes in favor of the spin variables $\theta_{s}$ described by Eq. (11). Note that the prefactor $\left(\frac{J_{1}}{m \omega_{0}^{2} a^{2}}\right) \sim \frac{v_{s}}{v_{c}} \sim \frac{E_{\text {spin }}}{E_{\text {charge }}}$ of the $2 k_{F}$ piece is nonuniversal and depends on the strength of the interactions. ${ }^{19}$ Since the spin-incoherent regime requires $E_{\text {spin }} \ll E_{\text {charge }}$, the $2 k_{F}$ oscillations are evidently weak even at zero temperature.

\section{D. $2 k_{F}$ density correlations}

The $2 k_{F}$ part of the density correlations (which show the most important temperature dependence for spinincoherent effects) can be computed in a straightforward way for $k_{B} T \ll E_{\text {spin }}$ using Eqs. (8) and (11) (Fiete et al., 2006; Fiete and Kindermann, 2007; Iucci et al., 2007). The main result is that these correlations are exponentially suppressed when $k_{B} T \gtrsim E_{\mathrm{spin}}$. Therefore in order to

\footnotetext{
${ }^{19}$ The prefactor of the $2 k_{F}$ contribution to Eq. (38) may depend also on the doping as explicitly seen in the Hubbard model (Giamarchi, 2004).
}

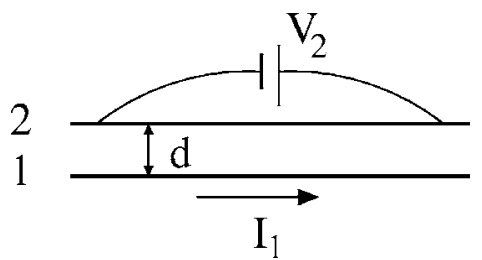

FIG. 8. Coulomb drag schematic. Two quantum wires are arranged parallel to one another and separated by a distance $d$. A dc current $I_{1}$ flows in the active wire 1 and a voltage bias $V_{2}$ is measured in the passive wire 2 when $I_{2}=0$.

understand the effects of spin-incoherent physics one may compute the $T=0$ result within the low-energy theory (8) and (11) to determine what will be missing when $k_{B} T \geqslant E_{\text {spin. }}{ }^{20}$

\section{E. Coulomb drag, noise, and dephasing}

In this subsection we discuss ways that the loss of $2 k_{F}$ oscillations may be observed in experiment. Spinincoherent effects are revealed through the temperature dependence of the $2 k_{F}$ density correlations in (i) Coulomb drag between quantum wires, (ii) the voltage noise on a gate close to a wire, and (iii) the dephasing time of a qubit near a wire. In many cases, unusual nonmonotonic temperature dependence may result providing a clear signature of the spin-incoherent Luttinger liquid.

In the Coulomb drag experiment (see Fig. 8) a current $I_{1}$ is driven in an active wire while a voltage bias $V_{2}$ is measured in a passive wire. The drag resistivity is defined as $r_{D}=-\lim _{I_{1} \rightarrow 0} \frac{e^{2}}{h} \frac{d V_{2}}{d I_{1}}$ and can be expressed (Zheng and MacDonald, 1993; Pustilnik et al., 2003) in terms of the imaginary part of the Fourier-transformed retarded density-density correlation function $\chi^{R}(k, \omega)$ computed from Eq. (38) (Fiete et al., 2006),

$$
r_{D}=\int_{0}^{\infty} d k \int_{0}^{\infty} d \omega \frac{k^{2} \tilde{U}_{12}^{2}(k)}{4 \pi^{2} n_{1} n_{2} T} \frac{\operatorname{Im} \chi_{1}^{R}(k, \omega) \operatorname{Im} \chi_{2}^{R}(k, \omega)}{\sinh ^{2}(\omega / 2 T)},
$$

where $n_{i}$ is the density of wire $i$ and $\tilde{U}_{12}(k)$ is the Fourier transform of the interwire interaction. The structure of Eq. (38) leads to $\chi(k, \omega) \approx \chi^{k \approx 0}(k, \omega)+\chi^{2 k_{F}}(k, \omega)$ $+\chi^{4 k_{F}}(k, \omega)$, so that the drag resistivity results from a sum of three terms. With a harmonic charge theory (8), the $k \approx 0$ contribution generically vanishes (Pustilnik et al., 2003; Fiete et al., 2006) leaving the drag to be dominated by the $2 k_{F}$ and $4 k_{F}$ pieces of $\chi(k, \omega)$. Since $\tilde{U}_{12}\left(2 k_{F}\right) \gg \tilde{U}_{12}\left(4 k_{F}\right)$ when $k_{F} d>1$, it is possible for the drag to be determined by the $2 k_{F}$ contributions at low temperature. However, when $k_{B} T \gg E_{\text {spin }}$ these correlations will be absent leaving only a $4 k_{F}$ contribution to

\footnotetext{
${ }^{20}$ Interesting changes are also expected in the same temperature range for the momentum distribution (Cheianov et al., 2005).
} 


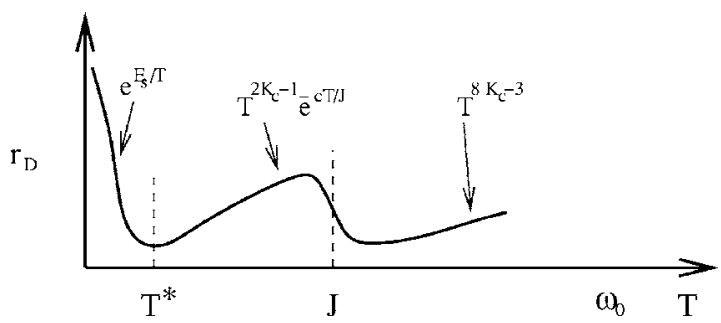

FIG. 9. Schematic of the possible temperature dependence of the Coulomb drag in identical wires of sufficiently low electron density that $J \ll E_{\text {charge }}=\hbar \omega_{0}$. The nonmonotonic temperature dependence shown can be obtained for $K_{c}>1 / 2$ and $\tilde{U}\left(4 k_{F}\right)$ $\ll \tilde{U}\left(2 k_{F}\right)$ which may be realized when $k_{F} d>1$. The temperature $T^{*}$ is the locking temperature of two identical wires, below which the drag exhibits activated behavior and $E_{S}$ is an energy gap associated with the locking. When $J \ll E_{\text {charge }}$ a sharp drop in the drag resistance should be observable for $k_{B} T \sim J$.

the drag. The resulting temperature dependence is shown in Fig. 9 (Fiete et al., 2006).

If a metallic gate is placed in proximity to a quantum wire in which a finite current $I$ is driven, there will be frequency-dependent voltage noise given by $S_{Q}(\omega)$ $=\frac{1}{2} \int d t e^{i \omega t}\langle\{V(t), V(0)\}\rangle$, where $V(t)$ is the voltage on the gate. Relating the voltage fluctuations to the density fluctuations in the wire gives (Fiete and Kindermann, 2007)

$$
S_{Q}(\omega)=\int \frac{d q}{2 \pi}|u(q)|^{2} \frac{\chi(q, \omega)+\chi(q,-\omega)}{2},
$$

where $S_{Q}(\omega)=S_{Q}^{q \approx 0}(\omega)+S_{Q}^{2 k_{F}}(\omega)+S_{Q}^{4 k_{F}}(\omega)$ from the structure of $\chi(q, \omega)$. Here $u(q)$ is the Fourier transform of the interaction between the gate and wire. The resulting voltage noise at zero temperature is (Fiete and Kindermann, 2007)

$$
\begin{aligned}
& S_{Q}^{q \approx 0}(\omega) \propto|u(0)|^{2}|\omega|, \\
& S_{Q}^{2 k_{F}}(\omega) \propto\left(\frac{J_{1}}{m \omega_{0}^{2} a^{2}}\right)^{2} \sum_{ \pm}\left|u\left(2 k_{F}\right)\right|^{2}\left|\omega \pm \frac{\omega_{I}}{2}\right|^{K_{s}+K_{c}-1}, \\
& S_{Q}^{4 k_{F}}(\omega) \propto \sum_{ \pm}\left|u\left(4 k_{F}\right)\right|^{2}\left|\omega \pm \omega_{I}\right|^{4 K_{c}-1}
\end{aligned}
$$

where $\omega_{I}=2 \pi I / e$. The frequency dependence of the voltage noise measured thus displays power-law singularities at the frequencies $\omega_{I} / 2$ and $\omega_{I}$ that are observable at low temperatures. The singularity in $S_{Q}^{2 k_{F}}$ at $\omega \approx \omega_{I} / 2$, however, becomes exponentially small as $k_{B} T \gtrsim J$ thus indicating an entry to the spin-incoherent regime. The dephasing time $\tau_{\varphi}$ of a qubit is determined by the zerofrequency part of the noise and is shown as a function of temperature in Fig. 10 (Fiete and Kindermann, 2007).

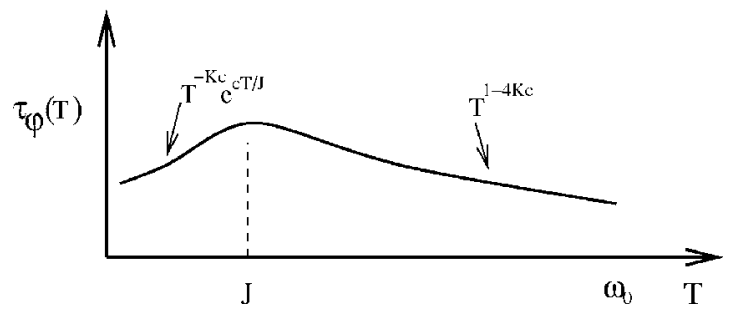

FIG. 10. Schematic temperature dependence of the dephasing time of a qubit. The constant $c$ in the exponential is $\mathcal{O}(1)$. The plot assumes $1 / 4<K_{c}<1$ and $K_{s}=1$.

\section{EXPERIMENTAL EVIDENCE FOR SPIN INCOHERENCE}

As the previous sections suggest, the theory of the spin-incoherent Luttinger liquid has developed rapidly in recent years. There are now many falsifiable predictions testable in experiment that are quite distinct from those of the low-temperature Luttinger liquid. On the experimental side much remains to be explored. So far, little directed effort has been made to investigate spinincoherent effects, but this situation is already changing rapidly.

At present there are strong indications in momentumresolved tunneling experiments on cleaved-edge overgrowth quantum wires (Auslaender et al., 2005; Steinberg et al., 2006) that the spin-incoherent regime $E_{\text {spin }}$ $\ll k_{B} T \ll E_{\text {charge }}$ is indeed obtained at large $r_{s}$ (small electron densities). Unfortunately, the analysis is somewhat involved (Fiete, Qian, et al., 2005; Steinberg et al., 2006). There is certainly urgent need for experiments that probe the spin-incoherent Luttinger liquid in more direct ways. The goal in this section is to present experimental evidence that this state is indeed reached in high quality ballistic semiconductor quantum wires at low particle density. $^{21}$

\section{A. Momentum-resolved tunneling at low particle density}

In many tunneling experiments, such as those done with a scanning tunneling microscope (STM), the tunneling essentially occurs at a single point-at the STM tip. For an STM, it can be shown that the tunneling conductance is related to the local density of states of the surface (Tersoff and Hamann, 1985; Fiete and Heller, 2003). For a fixed tip position, there is only energy resolution in the density of states. There is no momentum resolution because a local state must be built up of many different momentum states. Momentum-resolved tunneling is achieved when the tunneling occurs at many equivalent points, such as when there is translational symmetry in the system. This is precisely the case for tunneling be-

\footnotetext{
${ }^{21}$ Besides the momentum-resolved tunneling experiments, there is perhaps also the $0.7\left(2 e^{2} / h\right)$ conductance features in quantum point contacts that could be explained by spinincoherent effects in a finite length quantum wire attached to Fermi-liquid leads (Matveev, 2004a, 2004b).
} 


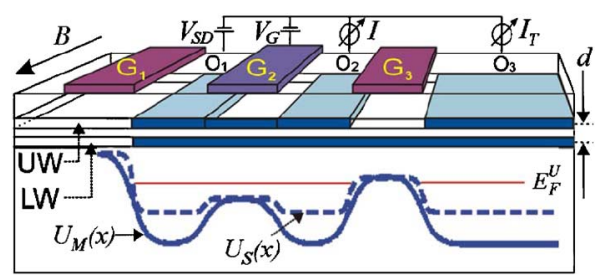

FIG. 11. (Color online) Schematic of the measurement setup with cleave plane front and perpendicular to the magnetic field $B$. The top gates $\left(G_{1}, G_{2}, G_{3}\right)$ are $2 \mu \mathrm{m}$ wide. The upper wire at the edge of the two-dimensional electron gas is $20 \mathrm{~nm}$ thick, the lower wire is $30 \mathrm{~nm}$ thick, and the barrier between them is 6-nm insulating AlGaAs. Here $U_{S}(x) / U_{M}(x)$ is the gateinduced potential for the single-mode/multimode wires, $E_{F}^{U}$ is the Fermi energy of the upper wire, $O_{1}$ is an Ohmic contact that serves as a source, and the Ohmic contacts $O_{2,3}$ serve as drains. The electron density in the quantum wires is modulated by a gate voltage $V_{G}$. The tunneling current is $I_{T}$ and the twoterminal current is $I$. From Steinberg et al., 2006.

tween two parallel quantum wires. The translational symmetry between parallel wires guarantees that momentum will be conserved when particles tunnel from one wire to the other. By applying a magnetic field perpendicular to the plane of the wires (as shown in Fig. 11) a tunable momentum boost proportional to the field strength and independent of the energy can be given to the tunneling particles. The energy, as with the STM, can be adjusted with a source-drain voltage bias. Thus momentum-resolved tunneling gives an additional "knob" (the momentum) to adjust when studying tunneling between two translationally invariant systems. As is well known (Mahan, 1990), to lowest order in the tunneling, the current is given by the spectral functions $A(k, \omega)$ of the two systems (the upper and lower wires in the present case). Therefore with independent control of both momentum and energy, the spectral functions of the double-wire system can be extracted. Having $A(k, \omega)$ in hand is tantamount to knowledge of the full dynamical properties and excitations of the system. This is just the information needed to observe dynamical effects such as spin-charge separation, and has in fact been observed in this type of setup (Auslaender et al., 2002, 2005).

A simple argument shows how the momentum boost occurs. Define a coordinate system so that the $x$ direction is parallel to the quantum wires (labeled by UW/LW for upper wire/lower wire) shown in Fig. 11. Let the $y$ direction be perpendicular to the $x$ direction and parallel to the plane containing the wires, and let $z$ be the direction of the magnetic field which is perpendicular to the plane of the wires. It is straightforward to see that $\vec{B}=\vec{\nabla} \times \vec{A}$, where $\vec{A}=(0, B x, 0)$. Consider an electron moving in the lower wire with a wave function proportional to $e^{i k x}$ that tunnels to the upper wire. After tunneling in the presence of the magnetic field it will pick up an Ahronov-Bohm type phase equal to $\frac{e}{\hbar c} \int \vec{A} \cdot d \vec{l}$ $=\frac{e}{\hbar c} \int_{0}^{d} B x d y=q_{B} x$, where $q_{B}=\frac{e B d}{\hbar c}$ is the momentum boost and $d$ is the center-to-center distance between the quan-

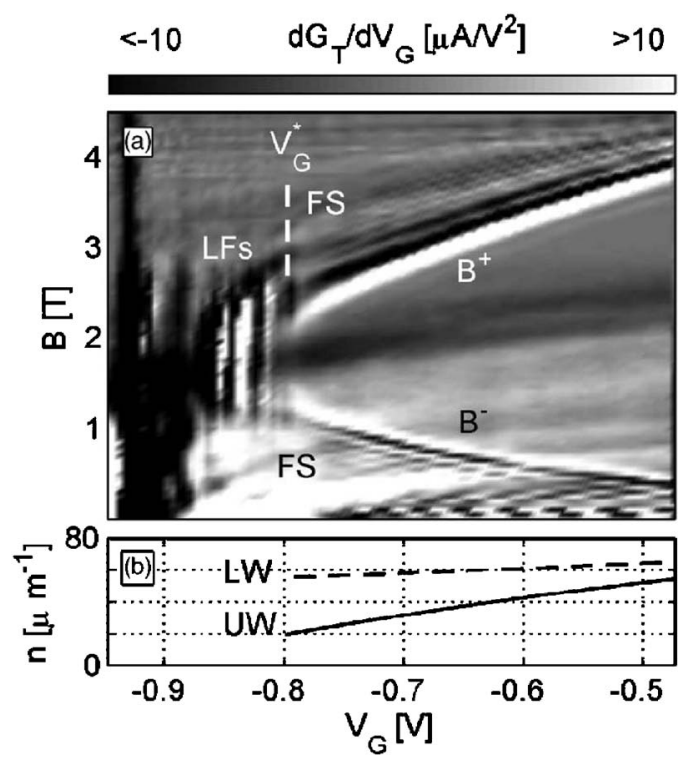

FIG. 12. Experimental data indicating a localization transition to a strongly correlated regime at low particle density. (a) Plot of $d G_{T} / d V_{G}$ vs $V_{G}$ and $B$ for a single-mode quantum wire. The applied bias $V_{S D}=100 \mu \mathrm{V}$ is selected to avoid the zerobias anomaly, but is still small enough to allow tunneling predominantly between the Fermi points of both wires. The upper and lower curves are momentum-conserving tunneling features $B^{ \pm}\left(V_{G}\right)$ and each curve is accompanied by finite-size features marked by FS. At low densities localized features (LFs) appear instead of these curves. The localization transition occurs at $V_{G}^{*}$. (b) indicates the upper wire (UW) and lower wire (LW) densities extracted from the data. From Steinberg et al., 2006.

tum wires as shown in Fig. 11. Thus upon tunneling the state $e^{i k x} \rightarrow e^{i\left(k+q_{B}\right) x}$, which looks like a momentum boost. As claimed earlier, the momentum boost is proportional to the magnetic field and independent of the energy. For an interacting system in which the eigenstates are not plane waves, the eigenstates may still be expressed as a linear combination of plane-wave states. Since the momentum boost $q_{B}$ is independent of $k$, each plane-wave state will have the same boost so that the overall state will have the same boost. Therefore regardless of the electron state in the upper and lower wires there will be a momentum boost of $q_{B}$ upon tunneling.

The type of experimental data that indicates the spinincoherent physics is shown in Fig. 12. To acquire these data the source drain voltage is set to a fixed value, $V_{S D}=100 \mu \mathrm{V}$, which is small enough to keep the tunneling in the linear-response regime. The tunneling conductance $G_{T}$ is then measured as a function of the perpendicular magnetic field $B$ and the voltage $V_{G}$ which modulates the voltage on gate $G_{2}$ shown in Fig. 11. When the gate $G_{2}$ is not grounded, the upper wire is effectively divided into three regions: (i) the region between the right edge of $G_{1}$ and the left edge of $G_{2}$, (ii) the region directly underneath $G_{2}$, and (iii) the region between the right edge of $G_{2}$ and the left edge of $G_{3}$. Because all regions of the upper wire are biased by $V_{S D}$ relative to the lower wire, electrons tunnel from all three 
regions. The main goal of the experiments reported by Steinberg et al. (2006) was to study the low-density regime of the quantum wires by applying a large negative bias to $G_{2}$. This creates an effective potential $U_{M / S}(x)$ shown schematically in Fig. 11 that leads to a lowdensity region in the center of the upper wire. In order to isolate the contribution to tunneling of this central, low-density region of the upper wire an effort was made to subtract off the contributions from the two higher density end regions. Under the assumption that the end regions are unaffected by small changes in $V_{G}$, the tunneling contribution from electrons in the central portion can be isolated by examining the change in $G_{T}$ with $V_{G}$, that is $d G_{T} / d V_{G}$. These types of data are shown in Fig. 12. A brighter signal indicates larger tunneling.

There are several important features of the data presented in Fig. 12. First, for $V_{G}>V_{G}^{*}$ there are two values of magnetic field, $B^{+}$and $B^{-}$, at any particular $V_{G}$, where the largest tunneling occurs. This is the expected result for electrons which are delocalized and freely propagating. For example, it can be shown (Auslaender et al., 2005; Steinberg et al., 2006) that the physics of the $B^{-}$ signal is electrons tunneling from right (left) moving states in one wire to right (left) moving states in the other. In other words, they are forward tunneling events. On the other hand, the $B^{+}$signal corresponds to electrons tunneling from right (left) moving states in one wire to left (right) moving states in the other, i.e., backward tunneling events. In order for this forward and backward interpretation to make sense, the electronic states must be reasonably close to states where momentum is a good quantum number, as it is for free electrons. The sharpness of the $B^{-}$and $B^{+}$for $V_{G}>V_{G}^{*}$ indicates that this is in fact the case. ${ }^{22}$

The second remarkable feature is the behavior of the data for $V_{G}<V_{G}^{*}$. Over this range of gate voltages the two distinct $B^{-}$and $B^{+}$features disappear and are replaced by very broad (in magnetic field) features. These broad features, called localized features (LFs) in Fig. 12, are also discrete in gate voltage indicating the onset of Coulomb blockade behavior below $V_{G}^{*}$. As shown in Sec. V.B, the localized features have an interpretation in terms of strong Wigner solidlike correlations and excited spin degrees of freedom.

The localized features are shown in more detail in Fig. 13(a). The intensity of the localized features can be quantified by the "weight" $\Gamma$ under a given localized feature for a specified $B$ value (Steinberg et al., 2006). ${ }^{23}$ The weight $\Gamma$ is shown in Fig. 13(c) and the temperature in

\footnotetext{
${ }^{22}$ This is not to say that the system is not a Luttinger liquid at these values of $V_{G}$. In fact, analysis of the data for these $V_{G}$ values show clear signatures of spin-charge separation and other Luttinger liquid effects (Auslaender et al., 2005). The spin and charge velocities can even be determined as a function of electron density in the wires.

${ }^{23}$ As explained (Steinberg et al., 2006), $\Gamma$ and $T$ are fit from the Coulomb blockade peaks to $\partial^{2} f / \partial V_{G}^{2}=\left(e^{2} / h\right) \Gamma \pi /$ $4 k_{B} T^{2} \tanh (x) \cosh ^{2}(x)$, where $x=(\epsilon-\mu) / k_{B} T$.
}

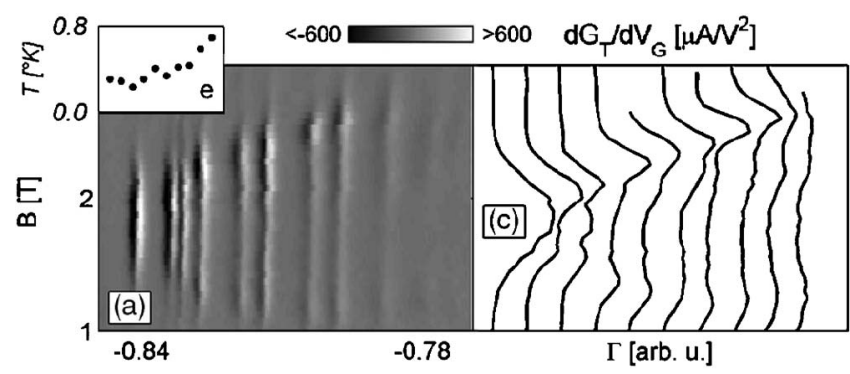

FIG. 13. Detail of the localization features in the strongly correlated regime at low particle density. (a) A high-resolution measurement of $d G_{T} / d V_{G}$ of localization features for a singlemode wire, $V_{S D}=50 \mu \mathrm{V}, d V_{G}=300 \mu \mathrm{V}$. (e) $T(N)$ of (a) where $N$ is the number of added electrons for each LF. (c) $\Gamma(B)$ $\propto|\Psi(k)|^{2}$. $\Gamma$ and $T$ are defined in the text. From Steinberg et al., 2006.

Fig. 13(e). The crucial feature for us is the double-lobed structure of $\Gamma$ as a function of $B$.

Before turning to the theory of these experiments, it is important to emphasize the features of the data in Fig. 13. First, for gate voltages more negative than those shown no more peaks appear. This indicates that for voltages just slightly larger (to the right) than the first peak there is one electron in the central region of the wire. Likewise, for voltages just larger than the value of the second peak, there are two electrons in the central portion of the wire, and so on. Second, the integrated (over $B$ ) weight under the peaks tends to decrease as the particle number increases. As discussed in the next subsection, this effect is related to the orthogonality catastrophe. Third, for the second peak and higher, $\Gamma$ has a double-lobed structure. The data are slightly asymmetric, and this is probably related to an asymmetric effect of $G_{2}$ on the electron density in the central region of the upper wire.

It is the details ${ }^{24}$ of the double-lobed $\Gamma$ that reveal the likelihood that $E_{\text {spin }} \ll k_{B} T \ll E_{\text {charge }}$ is reached at the low-particle (density) limit in the quantum wires. The most important features of the double-lobed structure are that the separation of the peaks grows with particle number, the widths of the peaks are comparable to the separation between them, and the center dip between peaks is shallower than the tails off to the sides. That the spacing and widths of the peaks track the particle number smoothly indicates that a collective, many-body effect is responsible for the shape. This behavior would not occur if electrons were all falling into distinct local minima. It turns out that the separation between peaks is related to the $k_{F}$ of the upper wire, and the widths of peaks as well as the shallow dip in between them all

\footnotetext{
${ }^{24}$ This reliance on details is the primary reason why more direct experimental probes of the spin-incoherent regime are needed. The theory is now well developed to predict many signatures in a variety of different experiments. It is hoped that this Colloquium will motivate such experiments to explore this regime of strongly interacting one-dimensional systems.
} 


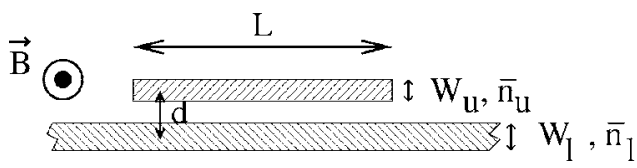

FIG. 14. Schematic geometry of electron tunneling between two parallel quantum wires, as in Fig. 11. Electrons are assumed to tunnel between an infinitely long lower wire and a short upper wire of length $L$. The wires are separated by a center-to-center distance $d$. The upper (lower) wire has a width $W_{u}\left(W_{l}\right)$ and an average electron density $\bar{n}_{u}\left(\bar{n}_{l}\right)$.

have a very natural interpretation in terms of a fluctuating Wigner solid model with highly excited spin degrees of freedom. It is the purpose of the next subsection to support this claim with direct theory that quantitatively suggests a fluctuating Wigner solid model is reasonable and $E_{\text {spin }} \ll k_{B} T$ is indeed reached for the relevant experimental parameters.

\section{B. Theoretical support for the spin-incoherent Luttinger liquid in experiment}

The main objective of this section is to provide theoretical support for the claim that a fluctuating Wigner solid model with thermally excited spins can explain the details of the double-lobed structure of $\Gamma(B)$ in Fig. 13(c). In order to avoid complicating our discussion of the data any more than necessary we consider a situation simpler than the one shown in Fig. 11. Our model isolates the central, low-density region of the upper wire just under $G_{2}$ and neglects the two ends between the gates $G_{1}$ and $G_{3}$. This geometry is shown in Fig. 14 .

\section{Exact diagonalization for few electrons}

As a first step towards quantitatively understanding the experiments of Steinberg et al. (2006), one would like to accurately study the few electron limit of the upper wire. Fiete, Qian, et al. (2005) carried out exact diagonalizations of four electrons in the upper wire assuming the geometry in Fig. 14, but using realistic interactions that took into account the widths of the quantum wires $W_{u} / W_{l}$, the separation between them $d$, and the dielectric constant of the material. The confining potential of the upper wire was assumed to be infinite at $x=0$ and $x=L$. The results for the ground-state density are shown in Fig. 15. Note the physical density dependence of the oscillations with position $x$ along with wire. For $n$ $\approx 10 e / \mu$ there is a transition from predominantly $2 k_{F}$ oscillations at higher densities to predominantly $4 k_{F}$ oscillations at lower densities. In fact, for $n \approx 1 e / \mu$ one can essentially see where the four electrons are sitting along with wire. What these exact numerics tell us is that for densities lower than $n \approx 10 e / \mu$ the ground-state electron density starts to look very much like that of a Wigner solid. Returning for a moment to the experiments of Steinberg et al. (2006), the best estimate of the density at which the localization transition occurs is $n$ $\approx(10-20) e / \mu$. Taken together, these results suggest that

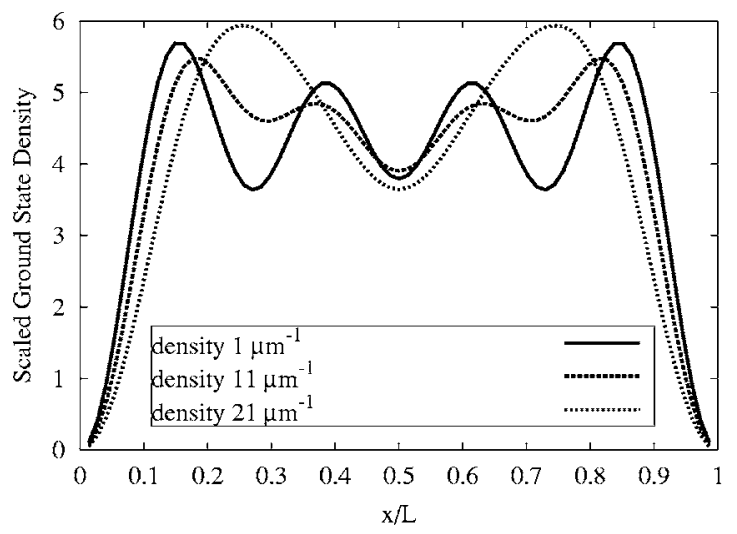

FIG. 15. Ground-state density of a four-electron system with spin. Scaled densities are measured in units of $1 / L$. In the label of the individual plot, density means the average physical density $N / L$ of the system, in units of $\mu \mathrm{m}^{-1}$.

the transition may be associated with a tendency towards strong Wigner solid correlations in the depleted region of the upper wire. The gate voltage dependence of the peaks in $\Gamma(B)$ shown in Fig. 13(c) are inconsistent with electrons falling into distinct local minima.

Within the exact diagonalizations for the four-electron system, the spin states can also be studied. As expected from the Lieb-Mattis theorem (Lieb and Mattis, 1962), the ground state is antiferromagnetically ordered. In the low-density limit, the numerical results showed (Fiete, Qian, et al., 2005) that the spin system can be approximated by the Heisenberg spin chain, Eq. (7). The spin excitation energies can also be studied by flipping one spin away from the antiferromagnetic configuration and asking how the energy cost depends on the density of electrons. From this, the nearest-neighbor exchange $J$ can be computed. The results are shown in Fig. 16. Returning again to experiment, for densities $n$ $\lesssim(10-20) e / \mu$ the upper wire is in the localized regime and characterized by strong Wigner solid correlations. It

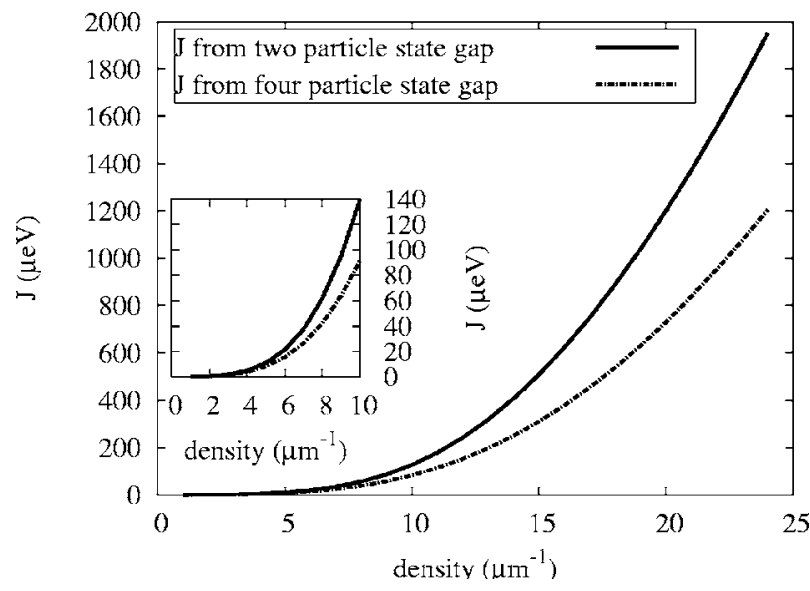

FIG. 16. Estimate of the spin-exchange energy $J$ from a twoand four-electron system as a function of density. Typical experimental temperatures $\left(T_{\exp }\right)$ are in the range $250 \mathrm{mK}-2 \mathrm{~K}$ $\approx 25-200 \mu \mathrm{eV}$. 
is useful to compare the computed exchange energy $J$ with the temperature of the experiment, $T_{\text {exp }}$. One finds that for $n \lesssim 10 e / \mu, J<k_{B} T_{\text {exp }}$, suggesting that at the lowest densities the spin-incoherent regime should be accessible.

So far, the numerics have provided evidence that near the localization transition Wigner solidlike correlations are developing and the magnetic exchange energy may be of order of the experimental temperature or smaller, opening the possibility of a description in terms of the model introduced in Sec. II. While these estimates suggest the possibility of the spin-incoherent regime at the lowest densities, the strongest evidence comes from the detailed line shape of $\Gamma(B)$. We therefore require a theory for this quantity.

The experimental results in Fig. 12(b) indicate that the gate $G_{2}$ preferentially depletes the upper wire, leaving the density of the lower wire relatively unchanged and also with a much larger density relative to the upper wire. Since at zero gate voltage both the upper and lower wires are in the high-density regime where interactions are less important, we can make an approximation that treats the lower wire as noninteracting throughout the full range of $V_{G}$. In this approximation (Fiete, Qian, et al., 2005), the tunneling conductance on the Coulomb blockade peaks ${ }^{25}$ is $G_{T}=G_{T}^{+}+G_{T}^{-}$where $G_{T}^{ \pm} \propto \mathcal{B}\left(k^{ \pm}\right)$with

$$
\mathcal{B}(k(B))=\sum_{\alpha \gamma \sigma}\left|\left\langle\Psi_{\alpha}^{N}\left|c_{k(B) \sigma}^{\dagger}\right| \Psi_{\gamma}^{N-1}\right\rangle\right|^{2} .
$$

Here $\Psi_{\alpha}^{N}$ is an $N$-particle eigenstate of the upper wire with quantum numbers $\alpha$ (perhaps the total spin and $z$ component of the spin), $c_{k \sigma}^{\dagger}$ creates a state with wave vector $k$ and $z$ component of the spin $\sigma$, and $k^{ \pm}(B)$ $= \pm k_{F}^{l}+e B d / \hbar c$, where $k_{F}^{l}$ is the Fermi wave vector of the lower wire. The result (42) tells us that the tunneling conductance on the localized feature is proportional to the overlap of an $N$-particle eigenstate with a state which is an $(N-1)$-particle eigenstate plus a plane-wave state. If we define

$$
M(k(B))=\left\langle\Psi_{\alpha}^{N}\left|c_{k(B) \sigma}^{\dagger}\right| \Psi_{\gamma}^{N-1}\right\rangle,
$$

then $M(k)$ can be expressed as the Fourier transform of a quasi wave function,

$$
M(k(B))=\int d x e^{i k(B) x} \Psi_{\mathrm{eff}}^{N^{*}}(x) / \sqrt{L},
$$

where $\Psi_{\text {eff }}^{N^{*}}(x)=\left\langle\Psi_{\alpha}^{N}\left|\psi_{\sigma}(x)\right| \Psi_{\gamma}^{N-1}\right\rangle$. Therefore the magnetic-field dependence of the conductance on the Coulomb blockage peaks reveals the Fourier transform of an effective wave function (Steinberg et al., 2006).

The Fourier-transform picture gives a satisfying interpretation of the momentum (magnetic-field) structure and the orthogonality catastrophe. If electrons were

\footnotetext{
${ }^{25}$ In Coulomb blockade theory tunneling occurs when there is a degeneracy of an $N-1$ and $N$ particle state.
}

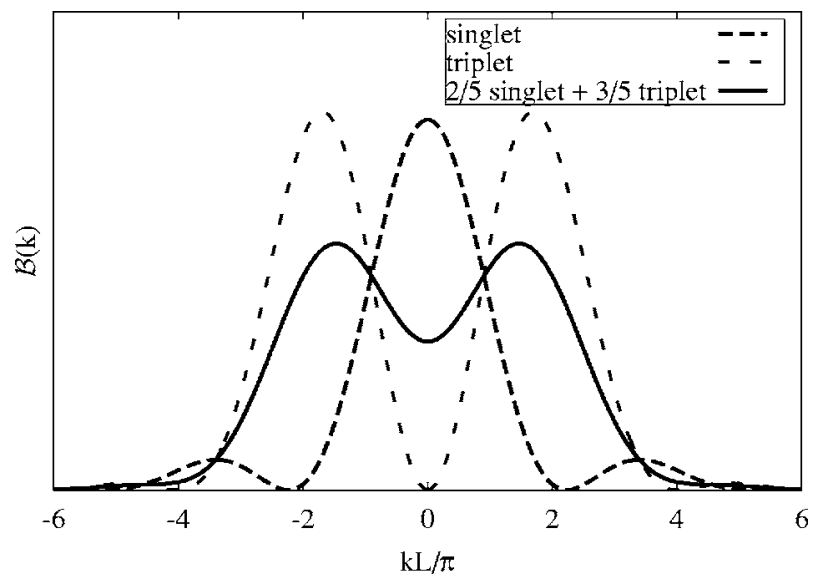

FIG. 17. Momentum dependence of tunneling conductance between one-electron and two-electron states for a wire of length $L=0.4 \mu \mathrm{m}$. The dashed curve shows the line shape $\left|M_{s}(k)\right|^{2}$ for tunneling into the singlet ground state of the twoparticle system. The short-dashed curve shows $\left|M_{t}(k)\right|^{2}$ for tunneling into the triplet ground state, applicable at $T=0$ when $E_{Z}>J$. The solid curve is a weighted average, applicable if $k_{B} T$ is large compared to both $E_{Z}$ and $J$ but small compared to the energy of the lowest charge excitation. The singlet + triplet line shape should be compared with the $\Gamma(B)$ line shape in Fig. 13(c).

noninteracting, there would be a peak at $\pm k_{F}^{u}$, with a width $\sim 1 / L$ due to the finite length of the system and the $B$-integrated weight would be independent of $N$. When interactions are present, adding a new electron shifts the states of all electrons previously in the system leading to an orthogonality catastrophe. This effect can explain the diminishing of the $B$-integrated weight of the localized features with increasing particle number $N$ in Figs. 13(a) and 13(c). The most crucial point is that the double-lobed structure with a shallow dip can be explained in terms of a strongly interacting state with highly thermally excited spin states. An illustrative example is the case of $N=2$. In this case, the ground state is a singlet. A singlet ground state will have a symmetric orbital part of the wave function. This leads to a maximum in $M(k)$ at $k=0$, as shown in Fig. 17. If the state the electron tunneled into was instead a triplet state, the orbital state is antisymmetric and this leads to a zero in $M(k)$ at $k=0$, as shown in Fig. 17. On the other hand, if the temperature is large, both singlet and triplet states are energetically allowed and this leads to the doublelobed structure with a shallow dip in the middle. The singlet + triplet line shape in Fig. 17 should be compared with the $\Gamma(B)$ line shape in Fig. 13(c). As shown in the next subsection, this feature is generic to a fluctuating Wigner solid model with highly thermally excited spins, that is, a spin-incoherent Luttinger liquid.

\section{Comparison with spin-incoherent Luttinger liquid theory}

In this subsection, we show the finite-temperature momentum structure of $\mathcal{B}(k)$ in Fig. 17 is generic to the spin-incoherent Luttinger liquid whose effective Hamil- 


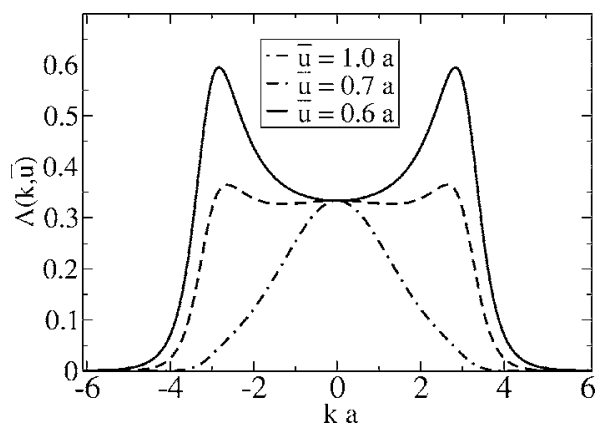

FIG. 18. Spectral function $A(k, \bar{u})$, which determines the momentum dependence of tunneling in the spin-incoherent regime. The quantity $\bar{u}$ is the root-mean-square electron displacement, due to quantum fluctuations, from the sites of a classical Wigner crystal, and $a$ is the lattice spacing. When $\bar{u}$ $\gtrsim a$, the momentum distribution is single lobed and peaked about zero momentum. In the opposite limit, when $\bar{u} \ll a$, the momentum distribution exhibits a doubled-lobed structure with peaks near $k= \pm 2 k_{F}$.

tonian is Eq. (9). To do this we compute $\mathcal{B}(k)$ using the spin-incoherent Luttinger liquid theory. This requires computing $A(k, \omega)$ of the upper wire which, as already discussed, is determined from the Green's function $\mathcal{G}(x, \tau)$. Because we are interested in large $\tau$ (small energies) the Fourier transform will be dominated by contributions from $|x|<v_{c} \tau$. The dominant, short-distance, $x$ $\sim a$, correlations that determine the momentum composition of the Green's function should thus be correctly described by Eq. (20). The Fourier transform is (Fiete, Qian, et al., 2005)

$$
\mathcal{G}_{\sigma}(k, \tau) \sim a\left(\frac{a}{v_{c} \tau}\right)^{1 / 4 K_{c}} A(k, \bar{u}(\tau)),
$$

where

$$
A(k, \bar{u}) \equiv e^{-k^{2} \bar{u}^{2} / 2}\left(\frac{3}{5+4 \cos (k a)}\right),
$$

and $\bar{u}(\tau)=\frac{a}{\pi} \sqrt{\left\langle\Theta(\tau)^{2}\right\rangle}$ which saturates at $\bar{u}\left(\tau=L / v_{c}\right)$ $=\frac{a}{\pi} \sqrt{2 K_{c} \ln (L / a)}$ for long times in a finite system.

Equations (45) and (46) are the central results for the spin-incoherent Luttinger liquid and they have several features worth emphasizing. The first is the momentum structure: There is an exponential envelope centered about zero momentum $e^{-k^{2} \bar{u}^{2} / 2}$ whose width is given by the parameter $\bar{u}$ measuring the fluctuations of an electron's position. Larger fluctuations imply a more sharply peaked envelope in momentum space. This envelope multiplies another momentum-dependent function, which is sensitive to the mean spacing of electrons and has maxima at $k= \pm \pi / a= \pm 2 k_{F}$. Results for different $\bar{u}$ are shown in Fig. 18. These results should be compared with Fig. 13(c) and the finite temperature results (singlet+triplet) of Fig. 17. The double-peaked structure with the soft dip is robust provided the fluctuations $\bar{u}$ are smaller than the interparticle spacing $a$, i.e., the system is a fluctuating Wigner solid with highly excited spins.

\section{OUTLOOK AND OPEN ISSUES}

In this Colloquium, the reader has been introduced to the concept of the spin-incoherent Luttinger liquid and how this state of strongly interacting $1 \mathrm{D}$ systems fits in with the more familiar Luttinger liquid state. While there are some similarities, there are important differences which make it an intriguing field of study. Two different classes of correlation functions have been identified: (i) those derived from particle nonconserving operators, and (ii) those derived from particle conserving operators. In the first class, exponential decays in space and logarithmic dependence in time (and frequency) are ubiquitous features in the spin-incoherent regime. In the second class, the correlation functions can be mapped onto a spinless Luttinger liquid deep in the spinincoherent regime. However, these correlation functions show dramatic temperature dependence when $k_{B} T$ $\approx E_{\text {spin }}$ and we discussed how this appears in Coulomb drag experiments between quantum wires and voltage fluctuations on a metallic gate near a quantum wire.

A number of open theoretical issues remain. There are many details of the crossover from the Luttinger liquid regime to the spin-incoherent Luttinger liquid regime that are not well understood because they are difficult to address analytically. Numerical work is thus highly desirable and may provide useful results for directly comparing to experiments, especially those in the regime $k_{B} T \approx E_{\text {spin }}$ where spin-incoherent effects are not fully manifest. Another issue that has not been addressed is how spin-orbit effects modify the spin-charge coupling discussed here and the energy scale for the observation of spin-incoherent effects. The coupling of external magnetic fields to orbital degrees of freedom should also be investigated. There are also related systems, such as the edges of quantum Hall states that may exhibit "incoherent" effects, perhaps in a neutral sector of the edge modes. 1D cold atomic gases may also provide a realization of some of the physics discussed here.

On the experimental side, some of the best indications of the spin-incoherent Luttinger liquid in high quality quantum wires have been presented. Theoretical estimates of the relevant energy scales and direct calculation of observable quantities provide compelling evidence that the spin-incoherent Luttinger liquid has been observed in momentum-resolved tunneling. Unfortunately, the analysis relies on a fairly detailed study of the data. The theory has now been sufficiently developed that there are many more direct ways to probe the spinincoherent Luttinger liquid. Such experiments are what is most urgently needed in the field. It is hoped that experimentalists will take up the challenge. It will undoubtedly lead to many insights and results not yet anticipated.

\section{ACKNOWLEDGMENTS}

I am grateful and deeply indebted to all my collaborators on this subject: Ophir Auslaender, Leon Balents, Bert Halperin, Markus Kindermann, Karyn Le Hur, 
Jiang Qian, Hadar Steinberg, Yaroslav Tserkovnyak, Amir Yacoby, and to the countless others with whom I have had insightful discussions. Among them are Matthew Fisher, Akira Furusaki, Thierry Giamarchi, Leonid Glazman, and Kostya Matveev. This work was supported by NSF Grants No. PHY05-51164 and No. DMR04-57440, the Packard Foundation, and the Lee A. DuBridge Foundation. I am also grateful for the hospitality of the Aspen Center for Physics where part of this work was done.

\section{REFERENCES}

Auslaender, O. M., H. Steinberg, A. Yacoby, Y. Tserkovnyak, B. I. Halperin, K. W. Baldwin, L. N. Pfeiffer, and K. W. West, 2005, Science 308, 88.

Auslaender, O. M., A. Yacoby, R. de Picciotto, K. W. Baldwin, L. N. Pfeiffer, and K. W. West, 2002, Science 295, 825.

Berkovich, A., 1991, J. Phys. A 24, 1543.

Castella, H., 1996, Phys. Rev. B 54, 17422.

Castro Neto, A. H., and M. P. A. Fisher, 1996, Phys. Rev. B 53, 9713.

Chang, A. M., 2003, Rev. Mod. Phys. 75, 1449.

Cheianov, V. V., H. Smith, and M. B. Zvonarev, 2005, Phys. Rev. A 71, 033610.

Cheianov, V. V., and M. B. Zvonarev, 2004a, Phys. Rev. Lett. 92, 176401.

Cheianov, V. V., and M. B. Zvonarev, 2004b, J. Phys. A 37, 2261.

Cronenwett, S. M., H. J. Lynch, D. Goldhaber-Gordon, L. P. Kouwenhoven, C. M. Marcus, K. Hirose, N. S. Wingreen, and V. Umansky, 2002, Phys. Rev. Lett. 88, 226805.

Damle, K., and S. Sachdev, 1998, Phys. Rev. B 57, 8307.

Damle, K., and S. Sachdev, 2005, Phys. Rev. Lett. 95, 187201.

DiCarlo, L., Y. Zhang, D. T. McClure, D. J. Reilly, C. M. Marcus, L. N. Pfeiffer, and K. W. West, 2006, Phys. Rev. Lett. 97, 036810.

Fiete, G. A., 2006, Phys. Rev. Lett. 97, 256403.

Fiete, G. A., and L. Balents, 2004, Phys. Rev. Lett. 93, 226401. Fiete, G. A., and E. J. Heller, 2003, Rev. Mod. Phys. 75, 933.

Fiete, G. A., and M. Kindermann, 2007, Phys. Rev. B 75, 035336.

Fiete, G. A., K. Le Hur, and L. Balents, 2005, Phys. Rev. B 72, 125416.

Fiete, G. A., K. Le Hur, and L. Balents, 2006, Phys. Rev. B 73, 165104.

Fiete, G. A., J. Qian, Y. Tserkovnyak, and B. I. Halperin, 2005, Phys. Rev. B 72, 045315.

Fogler, M., 2005a, Phys. Rev. B 71, 161304(R).

Fogler, M., 2005b, Phys. Rev. Lett. 94, 056405.

Fogler, M., and E. Pivovarov, 2005, Phys. Rev. B 72, 195344.

Furusaki, A., 1997, Phys. Rev. B 56, 9352.

Furusaki, A., and N. Nagaosa, 1993, Phys. Rev. B 47, 4631.

Giamarchi, T., 2004, Quantum Physics in One Dimension (Clarendon Press, Oxford).

Glazman, L. I., I. M. Ruzin, and B. I. Shklovskii, 1992, Phys. Rev. B 45, 8454.

Gogolin, A. O., 1993, Phys. Rev. Lett. 71, 2995.
Gogolin, A. O., A. A. Nersesyan, and A. M. Tsvelik, 1998, Bosonization and Strongly Correlated Systems (Cambridge University Press, Cambridge, England).

Häusler, W., 1996, Z. Phys. B: Condens. Matter 99, 551.

Häusler, W., L. Kecke, and A. H. MacDonald, 2002, Phys. Rev. B 65, 085104.

Iucci, A., G. A. Fiete, and T. Giamarchi, 2007, Phys. Rev. B 75, 205116.

Kakashvili, P., and H. Johannesson, 2006, e-print arXiv:condmat/0605062.

Kane, C. L., and M. P. A. Fisher, 1992, Phys. Rev. Lett. 68, 1220.

Kindermann, M., 2007, e-print arXiv:cond-mat/0703034.

Kindermann, M., and P. W. Brouwer, 2006a, Phys. Rev. B 74, 115121.

Kindermann, M., and P. W. Brouwer, 2006b, Phys. Rev. B 74, 125309.

Kindermann, M., P. W. Brouwer, and A. J. Millis, 2006, Phys. Rev. Lett. 97, 036809.

Klironomos, A. D., R. R. Ramazashvili, and K. A. Matveev, 2005, Phys. Rev. B 72, 195343.

Kominik, A., R. Egger, and A. O. Gogolin, 1997, Phys. Rev. B 56, 1153.

Le Hur, K., 2006, Phys. Rev. B 74, 165104.

Lieb, E., and D. Mattis, 1962, Phys. Rev. 125, 164.

Mahan, G. D., 1990, Many-Particle Physics, 2nd ed. (Plenum, New York).

Maslov, D., and M. Stone, 1995, Phys. Rev. B 52, R5539.

Matveev, K. A., 2004a, Phys. Rev. Lett. 92, 106801.

Matveev, K. A., 2004b, Phys. Rev. B 70, 245319.

Matveev, K. A., A. Furusaki, and L. I. Glazman, 2007, Phys. Rev. Lett. 98, 096403.

Novikov, D. S., 2005a, Phys. Rev. Lett. 95, 066401.

Novikov, D. S., 2005b, Phys. Rev. B 72, 235428.

Ogata, M., and H. Shiba, 1990, Phys. Rev. B 41, 2326.

Prokof'ev, N. V., 1994, Phys. Rev. B 49, 2148.

Pustilnik, M., E. G. Mishchenko, L. I. Glazman, and A. V., Andreev, 2003, Phys. Rev. Lett. 91, 126805.

Rapp, A., and G. Zarand, 2006, Phys. Rev. B 74, 014433.

Sachdev, S., and K. Damle, 1997, Phys. Rev. Lett. 78, 943.

Safi, I., and H. J. Schulz, 1995, Phys. Rev. B 52, R17040.

Schulz, H. J., 1990, Phys. Rev. Lett. 64, 2831.

Schulz, H. J., 1993, Phys. Rev. Lett. 71, 1864.

Steinberg, H., O. M. Auslaender, A. Yacoby, J. Qian, G. A. Fiete, Y. Tserkovnyak, B. I. Halperin, K. W. Baldwin, L. N. Pfeiffer, and K. W. West, 2006, Phys. Rev. B 73, 113307.

Syljuasen, O. F., 2007, Phys. Rev. Lett. 98, 166401.

Tanatar, B., and D. M. Ceperley, 1989, Phys. Rev. B 39, 5005.

Tersoff, J., and D. R. Hamann, 1985, Phys. Rev. B 31, 805.

Thomas, K. J., J. T. Nicholls, M. Y. Simmons, M. Pepper, D. R. Mace, and D. A. Ritchie, 1996, Phys. Rev. Lett. 77, 135.

Tsukamoto, Y., T. Fujii, and N. Kawakami, 1998a, Phys. Rev. B 58, 3633.

Tsukamoto, Y., T. Fujii, and N. Kawakami, 1998b, Eur. Phys. J. B 5, 479.

Voit, J., 1995, Rep. Prog. Phys. 58, 977.

Vollhardt, D., 1984, Rev. Mod. Phys. 56, 99.

Zheng, L., and A. H. MacDonald, 1993, Phys. Rev. B 48, 8203. 